\title{
Carbon-Hydrogen Bond Activation with a Cyclometalated Zirconocene Hydride: Mechanistic Differences Between Arene and Alkane Reductive Elimination.
}

Wesley H. Bernskoetter, Jaime A. Pool, Emil Lobkovsky, and Paul J. Chirik*

$$
\text { pc92@cornell.edu }
$$

Department of Chemistry and Chemical Biology, Baker Laboratory, Cornell University, Ithaca NY 14853.

-- SUPPORTING INFORMATION -- 


\section{Table of Contents}

${ }^{1} \mathrm{H}$ NMR spectrum of 2 in benzene- $d_{6} \quad$ S3

$\begin{array}{ll}\text { Crystallographic data for } \mathbf{1} & \text { S4 }\end{array}$

$\begin{array}{ll}\text { Crystallographic data for } 5 & \text { S11 }\end{array}$

$\begin{array}{ll}\text { Crystallographic data for } 7 & \text { S16 }\end{array}$ 


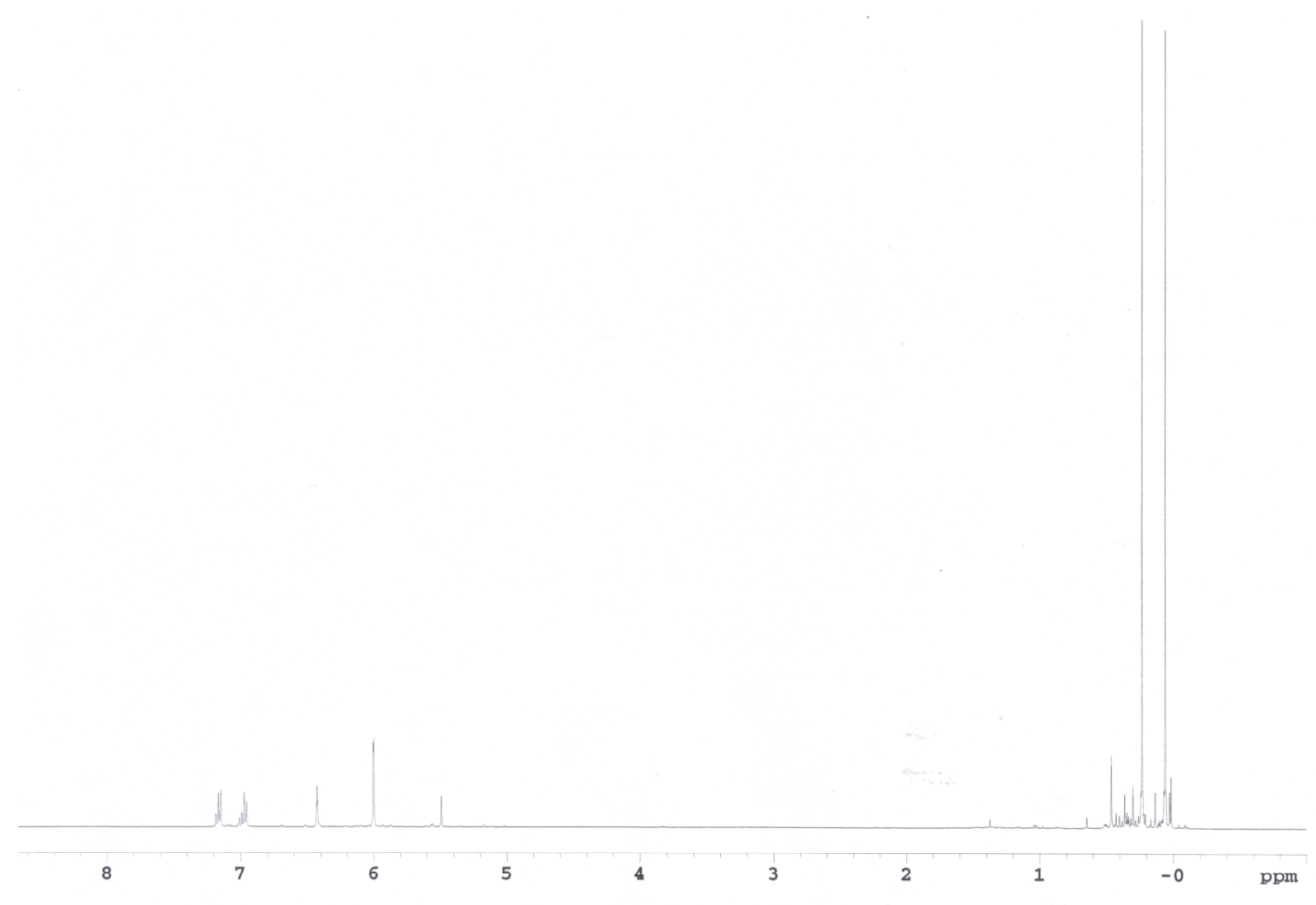

Figure S1. ${ }^{1} \mathrm{H}$ NMR spectrum of $\mathbf{2}$ in benzene- $d_{6}$. 


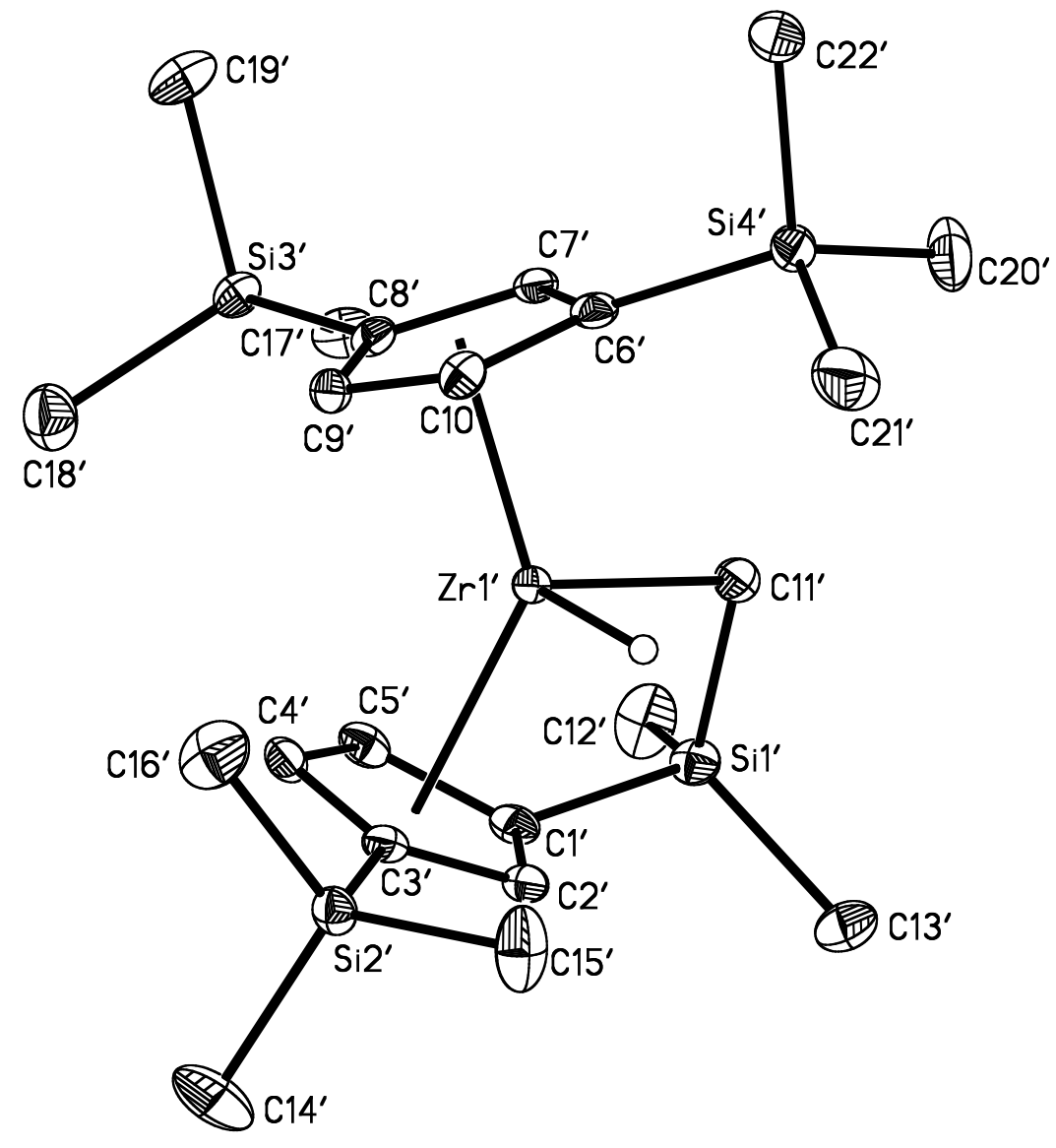

Figure S2. Fully labeled view of the molecular structure of $\mathbf{1}$ at $30 \%$ probability ellipsoids. Hydrogen atoms, except for the zirconium hydrides, omitted for clarity. 
Table S1. Crystal Data and Sstructure Refinement for $\mathbf{1}$

Identification code

$\mathrm{jd} 22$

Empirical formula

C22 H42 Si4 Zr

Formula weight

510.14

Temperature

173(2) K

Wavelength

$0.71073 \AA$

Crystal system

Monoclinic

Space group

Unit cell dimensions

$\mathrm{P} 2(1) / \mathrm{c}$

$$
\begin{array}{ll}
\mathrm{a}=12.4467(16) \AA & \alpha=90^{\circ} . \\
\mathrm{b}=13.9013(16) \AA & \beta=91.951(4)^{\circ} . \\
\mathrm{c}=32.399(4) \AA & \gamma=90^{\circ} .
\end{array}
$$

Volume

$5602.6(12) \AA^{3}$

$\mathrm{Z}$

8

Density (calculated)

$1.210 \mathrm{Mg} / \mathrm{m}^{3}$

Absorption coefficient

$0.570 \mathrm{~mm}^{-1}$

$\mathrm{F}(000)$

2160

Crystal size

$0.50 \times 0.30 \times 0.10 \mathrm{~mm}^{3}$

Theta range for data collection

1.64 to $26.37^{\circ}$.

Index ranges

$-14<=\mathrm{h}<=14,-15<=\mathrm{k}<=17,-39<=\mathrm{l}<=38$

Reflections collected

33716

Independent reflections

$10073[\mathrm{R}(\mathrm{int})=0.0393]$

Completeness to theta $=26.37^{\circ}$

Absorption correction

$87.9 \%$

\section{SADABS}

Max. and min. transmission

0.9452 and 0.7638

Refinement method

Full-matrix least-squares on $\mathrm{F}^{2}$

Data / restraints / parameters

10073 / 0 / 495

Goodness-of-fit on $\mathrm{F}^{2}$

1.203

Final $\mathrm{R}$ indices [I $>2 \operatorname{sigma}(\mathrm{I})]$

$\mathrm{R} 1=0.0599, \mathrm{wR} 2=0.1137$

$\mathrm{R}$ indices (all data)

$\mathrm{R} 1=0.0730, \mathrm{wR} 2=0.1178$

Largest diff. peak and hole 
Table S2. Bond Lengths $[\AA ̊]$ and Angles $\left[{ }^{\circ}\right]$ for $\mathbf{1}$.

\begin{tabular}{|c|c|c|c|}
\hline $\mathrm{Zr}(1)-\mathrm{H}(1 \mathrm{M})$ & $1.87(4)$ & $C(6)-C(7)$ & $1.425(5)$ \\
\hline $\operatorname{Zr}(1)-C(11)$ & $2.271(5)$ & $\mathrm{C}(7)-\mathrm{C}(8)$ & $1.422(5)$ \\
\hline $\operatorname{Zr}(1)-C(5)$ & $2.461(4)$ & $\mathrm{C}(8)-\mathrm{C}(9)$ & $1.429(6)$ \\
\hline $\mathrm{Zr}(1)-\mathrm{C}(1)$ & $2.485(4)$ & $\mathrm{C}(9)-\mathrm{C}(10)$ & $1.405(6)$ \\
\hline $\operatorname{Zr}(1)-C(9)$ & $2.495(4)$ & $\mathrm{Zr}\left(1^{\prime}\right)-\mathrm{H}\left(1 \mathrm{M}^{\prime}\right)$ & $1.78(5)$ \\
\hline $\mathrm{Zr}(1)-\mathrm{C}(2)$ & $2.508(4)$ & $\mathrm{Zr}\left(1^{\prime}\right)-\mathrm{C}\left(11^{\prime}\right)$ & $2.275(4)$ \\
\hline $\mathrm{Zr}(1)-\mathrm{C}(8)$ & $2.518(4)$ & $\operatorname{Zr}\left(1^{\prime}\right)-\mathrm{C}\left(5^{\prime}\right)$ & $2.466(4)$ \\
\hline $\mathrm{Zr}(1)-\mathrm{C}(4)$ & $2.525(4)$ & $\operatorname{Zr}\left(1^{\prime}\right)-\mathrm{C}\left(1^{\prime}\right)$ & $2.487(4)$ \\
\hline $\operatorname{Zr}(1)-C(10)$ & $2.531(4)$ & $\operatorname{Zr}\left(1^{\prime}\right)-\mathrm{C}\left(8^{\prime}\right)$ & $2.493(4)$ \\
\hline $\operatorname{Zr}(1)-C(7)$ & $2.538(4)$ & $\operatorname{Zr}\left(1^{\prime}\right)-\mathrm{C}\left(9^{\prime}\right)$ & $2.500(4)$ \\
\hline $\mathrm{Zr}(1)-\mathrm{C}(6)$ & $2.560(4)$ & $\operatorname{Zr}\left(1^{\prime}\right)-\mathrm{C}\left(2^{\prime}\right)$ & $2.499(4)$ \\
\hline $\mathrm{Zr}(1)-\mathrm{C}(3)$ & $2.576(4)$ & $\operatorname{Zr}\left(1^{\prime}\right)-\mathrm{C}\left(7^{\prime}\right)$ & $2.517(4)$ \\
\hline $\mathrm{Si}(1)-\mathrm{C}(11)$ & $1.849(5)$ & $\operatorname{Zr}\left(1^{\prime}\right)-\mathrm{C}\left(4^{\prime}\right)$ & $2.537(4)$ \\
\hline $\mathrm{Si}(1)-\mathrm{C}(12)$ & $1.860(5)$ & $\mathrm{Zr}\left(1^{\prime}\right)-\mathrm{C}\left(10^{\prime}\right)$ & $2.538(4)$ \\
\hline $\mathrm{Si}(1)-\mathrm{C}(13)$ & $1.863(5)$ & $\operatorname{Zr}\left(1^{\prime}\right)-\mathrm{C}\left(6^{\prime}\right)$ & $2.556(4)$ \\
\hline $\mathrm{Si}(1)-\mathrm{C}(1)$ & $1.881(4)$ & $\operatorname{Zr}\left(1^{\prime}\right)-\mathrm{C}\left(3^{\prime}\right)$ & $2.559(4)$ \\
\hline $\mathrm{Si}(2)-\mathrm{C}(3)$ & $1.858(4)$ & $\mathrm{Si}\left(1^{\prime}\right)-\mathrm{C}\left(11^{\prime}\right)$ & $1.840(4)$ \\
\hline $\mathrm{Si}(2)-\mathrm{C}(15)$ & $1.860(5)$ & $\mathrm{Si}\left(1^{\prime}\right)-\mathrm{C}\left(12^{\prime}\right)$ & $1.862(5)$ \\
\hline $\mathrm{Si}(2)-\mathrm{C}(16)$ & $1.860(5)$ & $\mathrm{Si}\left(1^{\prime}\right)-\mathrm{C}\left(13^{\prime}\right)$ & $1.864(5)$ \\
\hline $\mathrm{Si}(2)-\mathrm{C}(14)$ & $1.872(5)$ & $\operatorname{Si}\left(1^{\prime}\right)-C\left(1^{\prime}\right)$ & $1.885(4)$ \\
\hline $\mathrm{Si}(3)-\mathrm{C}(17)$ & $1.862(5)$ & $\mathrm{Si}\left(2^{\prime}\right)-\mathrm{C}\left(16^{\prime}\right)$ & $1.847(5)$ \\
\hline $\mathrm{Si}(3)-\mathrm{C}(18)$ & $1.862(4)$ & $\mathrm{Si}\left(2^{\prime}\right)-\mathrm{C}\left(15^{\prime}\right)$ & $1.858(5)$ \\
\hline $\mathrm{Si}(3)-\mathrm{C}(8)$ & $1.869(4)$ & $\mathrm{Si}\left(2^{\prime}\right)-\mathrm{C}\left(14^{\prime}\right)$ & $1.869(5)$ \\
\hline $\mathrm{Si}(3)-\mathrm{C}(19)$ & $1.872(5)$ & $\operatorname{Si}\left(2^{\prime}\right)-C\left(3^{\prime}\right)$ & $1.871(4)$ \\
\hline $\mathrm{Si}(4)-\mathrm{C}(20)$ & $1.857(5)$ & $\operatorname{Si}\left(3^{\prime}\right)-C\left(8^{\prime}\right)$ & $1.868(4)$ \\
\hline $\operatorname{Si}(4)-C(21)$ & $1.862(5)$ & $\operatorname{Si}\left(3^{\prime}\right)-C\left(17^{\prime}\right)$ & $1.869(5)$ \\
\hline $\mathrm{Si}(4)-\mathrm{C}(22)$ & $1.865(5)$ & $\mathrm{Si}\left(3^{\prime}\right)-\mathrm{C}\left(19^{\prime}\right)$ & $1.871(5)$ \\
\hline $\mathrm{Si}(4)-\mathrm{C}(6)$ & $1.873(4)$ & $\operatorname{Si}\left(3^{\prime}\right)-C\left(18^{\prime}\right)$ & $1.868(5)$ \\
\hline $\mathrm{C}(1)-\mathrm{C}(5)$ & $1.431(6)$ & $\operatorname{Si}\left(4^{\prime}\right)-C\left(20^{\prime}\right)$ & $1.859(5)$ \\
\hline $\mathrm{C}(1)-\mathrm{C}(2)$ & $1.420(6)$ & $\operatorname{Si}\left(4^{\prime}\right)-C\left(21^{\prime}\right)$ & $1.865(6)$ \\
\hline$C(2)-C(3)$ & $1.427(6)$ & $\operatorname{Si}\left(4^{\prime}\right)-C\left(22^{\prime}\right)$ & $1.866(5)$ \\
\hline$C(3)-C(4)$ & $1.423(6)$ & $\operatorname{Si}\left(4^{\prime}\right)-C\left(6^{\prime}\right)$ & $1.877(4)$ \\
\hline$C(4)-C(5)$ & $1.409(6)$ & $\mathrm{C}\left(1^{\prime}\right)-\mathrm{C}\left(2^{\prime}\right)$ & $1.418(6)$ \\
\hline$C(6)-C(10)$ & $1.422(6)$ & $C\left(1^{\prime}\right)-C\left(5^{\prime}\right)$ & $1.420(6)$ \\
\hline
\end{tabular}




\begin{tabular}{|c|c|c|c|}
\hline$C\left(2^{\prime}\right)-C\left(3^{\prime}\right)$ & $1.430(6)$ & $\mathrm{C}(8)-\mathrm{Zr}(1)-\mathrm{C}(4)$ & $89.75(13)$ \\
\hline$C\left(3^{\prime}\right)-C\left(4^{\prime}\right)$ & $1.408(6)$ & $\mathrm{H}(1 \mathrm{M})-\mathrm{Zr}(1)-\mathrm{C}(10)$ & $72.5(13)$ \\
\hline$C\left(4^{\prime}\right)-C\left(5^{\prime}\right)$ & $1.412(6)$ & $C(11)-\operatorname{Zr}(1)-C(10)$ & $123.86(15)$ \\
\hline $\mathrm{C}\left(6^{\prime}\right)-\mathrm{C}\left(7^{\prime}\right)$ & $1.416(6)$ & $C(5)-\operatorname{Zr}(1)-C(10)$ & $134.18(14)$ \\
\hline $\mathrm{C}\left(6^{\prime}\right)-\mathrm{C}\left(10^{\prime}\right)$ & $1.432(6)$ & $\mathrm{C}(1)-\operatorname{Zr}(1)-\mathrm{C}(10)$ & $165.21(14)$ \\
\hline $\mathrm{C}\left(7^{\prime}\right)-\mathrm{C}\left(8^{\prime}\right)$ & $1.426(6)$ & $\mathrm{C}(9)-\operatorname{Zr}(1)-\mathrm{C}(10)$ & $32.45(13)$ \\
\hline $\mathrm{C}\left(8^{\prime}\right)-\mathrm{C}\left(9^{\prime}\right)$ & $1.435(6)$ & $C(2)-\operatorname{Zr}(1)-C(10)$ & $140.12(14)$ \\
\hline \multirow[t]{2}{*}{$\mathrm{C}\left(9^{\prime}\right)-\mathrm{C}\left(10^{\prime}\right)$} & $1.393(6)$ & $\mathrm{C}(8)-\operatorname{Zr}(1)-\mathrm{C}(10)$ & $54.50(13)$ \\
\hline & & $\mathrm{C}(4)-\operatorname{Zr}(1)-\mathrm{C}(10)$ & $110.34(14)$ \\
\hline $\mathrm{H}(1 \mathrm{M})-\mathrm{Zr}(1)-\mathrm{C}(11)$ & $103.5(13)$ & $\mathrm{H}(1 \mathrm{M})-\mathrm{Zr}(1)-\mathrm{C}(7)$ & $116.3(13)$ \\
\hline $\mathrm{H}(1 \mathrm{M})-\mathrm{Zr}(1)-\mathrm{C}(5)$ & $128.7(13)$ & $\mathrm{C}(11)-\operatorname{Zr}(1)-\mathrm{C}(7)$ & $84.54(15)$ \\
\hline$C(11)-\operatorname{Zr}(1)-C(5)$ & $92.98(15)$ & $C(5)-\operatorname{Zr}(1)-C(7)$ & $113.40(13)$ \\
\hline $\mathrm{H}(1 \mathrm{M})-\mathrm{Zr}(1)-\mathrm{C}(1)$ & $108.8(13)$ & $C(1)-\operatorname{Zr}(1)-C(7)$ & $132.46(13)$ \\
\hline $\mathrm{C}(11)-\operatorname{Zr}(1)-\mathrm{C}(1)$ & $70.63(14)$ & $\mathrm{C}(9)-\mathrm{Zr}(1)-\mathrm{C}(7)$ & $53.50(13)$ \\
\hline$C(5)-Z r(1)-C(1)$ & $33.63(14)$ & $\mathrm{C}(2)-\mathrm{Zr}(1)-\mathrm{C}(7)$ & $165.47(13)$ \\
\hline $\mathrm{H}(1 \mathrm{M})-\mathrm{Zr}(1)-\mathrm{C}(9)$ & $97.1(13)$ & $\mathrm{C}(8)-\mathrm{Zr}(1)-\mathrm{C}(7)$ & $32.66(12)$ \\
\hline $\mathrm{C}(11)-\operatorname{Zr}(1)-\mathrm{C}(9)$ & $138.02(15)$ & $\mathrm{C}(4)-\mathrm{Zr}(1)-\mathrm{C}(7)$ & $121.75(13)$ \\
\hline $\mathrm{C}(5)-\mathrm{Zr}(1)-\mathrm{C}(9)$ & $102.07(14)$ & $\mathrm{C}(10)-\mathrm{Zr}(1)-\mathrm{C}(7)$ & $53.16(13)$ \\
\hline $\mathrm{C}(1)-\mathrm{Zr}(1)-\mathrm{C}(9)$ & $135.28(13)$ & $\mathrm{H}(1 \mathrm{M})-\mathrm{Zr}(1)-\mathrm{C}(6)$ & $83.8(13)$ \\
\hline $\mathrm{H}(1 \mathrm{M})-\mathrm{Zr}(1)-\mathrm{C}(2)$ & $77.7(13)$ & $\mathrm{C}(11)-\mathrm{Zr}(1)-\mathrm{C}(6)$ & $91.95(15)$ \\
\hline$C(11)-\operatorname{Zr}(1)-C(2)$ & $88.34(15)$ & $C(5)-\operatorname{Zr}(1)-C(6)$ & $144.53(13)$ \\
\hline$C(5)-Z r(1)-C(2)$ & $54.26(14)$ & $\mathrm{C}(1)-\mathrm{Zr}(1)-\mathrm{C}(6)$ & $160.22(14)$ \\
\hline$C(1)-Z r(1)-C(2)$ & $33.04(14)$ & $C(9)-\operatorname{Zr}(1)-C(6)$ & $54.17(13)$ \\
\hline$C(9)-Z r(1)-C(2)$ & $132.05(13)$ & $\mathrm{C}(2)-\mathrm{Zr}(1)-\mathrm{C}(6)$ & $161.07(13)$ \\
\hline $\mathrm{H}(1 \mathrm{M})-\mathrm{Zr}(1)-\mathrm{C}(8)$ & $126.8(13)$ & $C(8)-\operatorname{Zr}(1)-C(6)$ & $54.92(12)$ \\
\hline $\mathrm{C}(11)-\operatorname{Zr}(1)-\mathrm{C}(8)$ & $109.23(15)$ & $\mathrm{C}(4)-\mathrm{Zr}(1)-\mathrm{C}(6)$ & 137.91(13) \\
\hline$C(5)-\operatorname{Zr}(1)-C(8)$ & $90.46(13)$ & $C(10)-\operatorname{Zr}(1)-C(6)$ & $32.44(13)$ \\
\hline $\mathrm{C}(1)-\mathrm{Zr}(1)-\mathrm{C}(8)$ & $121.00(13)$ & $C(7)-\operatorname{Zr}(1)-C(6)$ & $32.44(12)$ \\
\hline $\mathrm{C}(9)-\mathrm{Zr}(1)-\mathrm{C}(8)$ & $33.13(13)$ & $\mathrm{H}(1 \mathrm{M})-\mathrm{Zr}(1)-\mathrm{C}(3)$ & $75.5(13)$ \\
\hline$C(2)-Z r(1)-C(8)$ & $142.03(13)$ & $\mathrm{C}(11)-\mathrm{Zr}(1)-\mathrm{C}(3)$ & $120.68(15)$ \\
\hline $\mathrm{H}(1 \mathrm{M})-\mathrm{Zr}(1)-\mathrm{C}(4)$ & $105.4(13)$ & $C(5)-\operatorname{Zr}(1)-C(3)$ & $54.67(13)$ \\
\hline $\mathrm{C}(11)-\operatorname{Zr}(1)-\mathrm{C}(4)$ & $123.90(15)$ & $\mathrm{C}(1)-\mathrm{Zr}(1)-\mathrm{C}(3)$ & $55.29(13)$ \\
\hline$C(5)-\operatorname{Zr}(1)-C(4)$ & $32.79(14)$ & $C(9)-\operatorname{Zr}(1)-C(3)$ & $99.62(13)$ \\
\hline$C(1)-Z r(1)-C(4)$ & $54.88(14)$ & $\mathrm{C}(2)-\mathrm{Zr}(1)-\mathrm{C}(3)$ & $32.56(13)$ \\
\hline$C(9)-\operatorname{Zr}(1)-C(4)$ & $83.77(13)$ & $\mathrm{C}(8)-\mathrm{Zr}(1)-\mathrm{C}(3)$ & $117.90(13)$ \\
\hline$C(2)-Z r(1)-C(4)$ & $53.36(13)$ & $\mathrm{C}(4)-\mathrm{Zr}(1)-\mathrm{C}(3)$ & $32.37(13)$ \\
\hline
\end{tabular}




\begin{tabular}{|c|c|c|c|}
\hline $\mathrm{C}(10)-\mathrm{Zr}(1)-\mathrm{C}(3)$ & $112.45(14)$ & $\operatorname{Si}(1)-C(1)-\operatorname{Zr}(1)$ & $92.71(15)$ \\
\hline $\mathrm{C}(7)-\mathrm{Zr}(1)-\mathrm{C}(3)$ & $150.28(13)$ & $\mathrm{C}(1)-\mathrm{C}(2)-\mathrm{C}(3)$ & 111.2(4) \\
\hline$C(6)-\operatorname{Zr}(1)-C(3)$ & $144.43(14)$ & $\mathrm{C}(1)-\mathrm{C}(2)-\operatorname{Zr}(1)$ & $72.6(2)$ \\
\hline $\mathrm{C}(11)-\mathrm{Si}(1)-\mathrm{C}(12)$ & $113.6(2)$ & $\mathrm{C}(3)-\mathrm{C}(2)-\mathrm{Zr}(1)$ & $76.3(2)$ \\
\hline $\mathrm{C}(11)-\mathrm{Si}(1)-\mathrm{C}(13)$ & $116.4(2)$ & $\mathrm{C}(4)-\mathrm{C}(3)-\mathrm{C}(2)$ & $105.0(4)$ \\
\hline $\mathrm{C}(12)-\mathrm{Si}(1)-\mathrm{C}(13)$ & $110.7(2)$ & $\mathrm{C}(4)-\mathrm{C}(3)-\mathrm{Si}(2)$ & $127.5(3)$ \\
\hline $\mathrm{C}(11)-\mathrm{Si}(1)-\mathrm{C}(1)$ & $95.23(19)$ & $\mathrm{C}(2)-\mathrm{C}(3)-\mathrm{Si}(2)$ & $127.4(3)$ \\
\hline $\mathrm{C}(12)-\mathrm{Si}(1)-\mathrm{C}(1)$ & $108.7(2)$ & $\mathrm{C}(4)-\mathrm{C}(3)-\operatorname{Zr}(1)$ & $71.8(2)$ \\
\hline $\mathrm{C}(13)-\mathrm{Si}(1)-\mathrm{C}(1)$ & 111.2(2) & $C(2)-C(3)-\operatorname{Zr}(1)$ & 71.1(2) \\
\hline $\mathrm{C}(11)-\operatorname{Si}(1)-\operatorname{Zr}(1)$ & $44.41(14)$ & $\operatorname{Si}(2)-C(3)-\operatorname{Zr}(1)$ & 124.91(19) \\
\hline $\mathrm{C}(12)-\operatorname{Si}(1)-\operatorname{Zr}(1)$ & $117.86(17)$ & $\mathrm{C}(5)-\mathrm{C}(4)-\mathrm{C}(3)$ & $109.7(4)$ \\
\hline $\mathrm{C}(13)-\mathrm{Si}(1)-\operatorname{Zr}(1)$ & $131.44(17)$ & $C(5)-C(4)-\operatorname{Zr}(1)$ & 71.1(2) \\
\hline$C(1)-\operatorname{Si}(1)-\operatorname{Zr}(1)$ & $51.17(13)$ & $C(3)-C(4)-\operatorname{Zr}(1)$ & $75.8(2)$ \\
\hline$C(3)-S i(2)-C(15)$ & $108.9(2)$ & $\mathrm{C}(4)-\mathrm{C}(5)-\mathrm{C}(1)$ & $108.8(4)$ \\
\hline$C(3)-S i(2)-C(16)$ & $110.6(2)$ & $C(4)-C(5)-\operatorname{Zr}(1)$ & $76.1(2)$ \\
\hline $\mathrm{C}(15)-\mathrm{Si}(2)-\mathrm{C}(16)$ & 109.7(3) & $C(1)-C(5)-\operatorname{Zr}(1)$ & 74.1(2) \\
\hline$C(3)-S i(2)-C(14)$ & $108.4(2)$ & $\mathrm{C}(10)-\mathrm{C}(6)-\mathrm{C}(7)$ & $105.6(3)$ \\
\hline $\mathrm{C}(15)-\mathrm{Si}(2)-\mathrm{C}(14)$ & $110.4(2)$ & $\mathrm{C}(10)-\mathrm{C}(6)-\mathrm{Si}(4)$ & $127.2(3)$ \\
\hline $\mathrm{C}(16)-\mathrm{Si}(2)-\mathrm{C}(14)$ & $109.0(2)$ & $C(7)-C(6)-S i(4)$ & $126.9(3)$ \\
\hline $\mathrm{C}(17)-\mathrm{Si}(3)-\mathrm{C}(18)$ & $110.8(2)$ & $C(10)-C(6)-\operatorname{Zr}(1)$ & $72.6(2)$ \\
\hline $\mathrm{C}(17)-\mathrm{Si}(3)-\mathrm{C}(8)$ & 111.1(2) & $C(7)-C(6)-\operatorname{Zr}(1)$ & $72.9(2)$ \\
\hline $\mathrm{C}(18)-\mathrm{Si}(3)-\mathrm{C}(8)$ & 111.1(2) & $\mathrm{Si}(4)-\mathrm{C}(6)-\mathrm{Zr}(1)$ & $123.49(19)$ \\
\hline $\mathrm{C}(17)-\mathrm{Si}(3)-\mathrm{C}(19)$ & $108.4(2)$ & $C(6)-C(7)-C(8)$ & $110.7(4)$ \\
\hline $\mathrm{C}(18)-\mathrm{Si}(3)-\mathrm{C}(19)$ & $107.8(2)$ & $C(6)-C(7)-\operatorname{Zr}(1)$ & $74.6(2)$ \\
\hline$C(8)-\operatorname{Si}(3)-C(19)$ & $107.5(2)$ & $\mathrm{C}(8)-\mathrm{C}(7)-\operatorname{Zr}(1)$ & $72.9(2)$ \\
\hline $\mathrm{C}(20)-\mathrm{Si}(4)-\mathrm{C}(21)$ & 109.9(3) & $\mathrm{C}(7)-\mathrm{C}(8)-\mathrm{C}(9)$ & $105.2(3)$ \\
\hline $\mathrm{C}(20)-\mathrm{Si}(4)-\mathrm{C}(22)$ & $109.9(2)$ & $\mathrm{C}(7)-\mathrm{C}(8)-\mathrm{Si}(3)$ & $124.9(3)$ \\
\hline $\mathrm{C}(21)-\mathrm{Si}(4)-\mathrm{C}(22)$ & 109.1(3) & $\mathrm{C}(9)-\mathrm{C}(8)-\mathrm{Si}(3)$ & $127.6(3)$ \\
\hline$C(20)-S i(4)-C(6)$ & $109.8(2)$ & $\mathrm{C}(7)-\mathrm{C}(8)-\operatorname{Zr}(1)$ & $74.5(2)$ \\
\hline$C(21)-S i(4)-C(6)$ & $110.6(2)$ & $\mathrm{C}(9)-\mathrm{C}(8)-\operatorname{Zr}(1)$ & $72.5(2)$ \\
\hline$C(22)-S i(4)-C(6)$ & $107.5(2)$ & $\operatorname{Si}(3)-C(8)-\operatorname{Zr}(1)$ & $130.9(2)$ \\
\hline$C(5)-C(1)-C(2)$ & 105.3(4) & $\mathrm{C}(10)-\mathrm{C}(9)-\mathrm{C}(8)$ & 109.3(4) \\
\hline $\mathrm{C}(5)-\mathrm{C}(1)-\mathrm{Si}(1)$ & $123.0(3)$ & $C(10)-C(9)-Z r(1)$ & $75.2(2)$ \\
\hline$C(2)-C(1)-S i(1)$ & $123.4(3)$ & $\mathrm{C}(8)-\mathrm{C}(9)-\operatorname{Zr}(1)$ & $74.3(2)$ \\
\hline$C(5)-C(1)-\operatorname{Zr}(1)$ & $72.3(2)$ & $\mathrm{C}(9)-\mathrm{C}(10)-\mathrm{C}(6)$ & 109.1(4) \\
\hline$C(2)-C(1)-Z r(1)$ & $74.4(2)$ & $\mathrm{C}(9)-\mathrm{C}(10)-\mathrm{Zr}(1)$ & $72.3(2)$ \\
\hline
\end{tabular}




\begin{tabular}{|c|c|c|c|}
\hline$C(6)-C(10)-Z r(1)$ & $74.9(2)$ & $C\left(2^{\prime}\right)-\operatorname{Zr}\left(1^{\prime}\right)-C\left(4^{\prime}\right)$ & $53.24(15)$ \\
\hline $\mathrm{Si}(1)-\mathrm{C}(11)-\mathrm{Zr}(1)$ & $100.87(19)$ & $C\left(7^{\prime}\right)-\operatorname{Zr}\left(1^{\prime}\right)-C\left(4^{\prime}\right)$ & $124.65(14)$ \\
\hline $\mathrm{H}\left(1 \mathrm{M}^{\prime}\right)-\mathrm{Zr}\left(1^{\prime}\right)-\mathrm{C}\left(11^{\prime}\right)$ & $97.8(17)$ & $\mathrm{H}\left(1 \mathrm{M}^{\prime}\right)-\mathrm{Zr}\left(1^{\prime}\right)-\mathrm{C}\left(10^{\prime}\right)$ & $77.3(17)$ \\
\hline $\mathrm{H}\left(1 \mathrm{M}^{\prime}\right)-\mathrm{Zr}\left(1^{\prime}\right)-\mathrm{C}\left(5^{\prime}\right)$ & $128.8(17)$ & $\mathrm{C}\left(11^{\prime}\right)-\operatorname{Zr}\left(1^{\prime}\right)-\mathrm{C}\left(10^{\prime}\right)$ & $127.72(14)$ \\
\hline $\mathrm{C}\left(11^{\prime}\right)-\mathrm{Zr}\left(1^{\prime}\right)-\mathrm{C}\left(5^{\prime}\right)$ & $91.70(15)$ & $\mathrm{C}\left(5^{\prime}\right)-\mathrm{Zr}\left(1^{\prime}\right)-\mathrm{C}\left(10^{\prime}\right)$ & $132.10(14)$ \\
\hline $\mathrm{H}\left(1 \mathrm{M}^{\prime}\right)-\mathrm{Zr}\left(1^{\prime}\right)-\mathrm{C}\left(1^{\prime}\right)$ & $104.9(17)$ & $\mathrm{C}\left(1^{\prime}\right)-\mathrm{Zr}\left(1^{\prime}\right)-\mathrm{C}\left(10^{\prime}\right)$ & $161.38(13)$ \\
\hline$C\left(11^{\prime}\right)-\operatorname{Zr}\left(1^{\prime}\right)-C\left(1^{\prime}\right)$ & $70.71(14)$ & $\mathrm{C}\left(8^{\prime}\right)-\operatorname{Zr}\left(1^{\prime}\right)-\mathrm{C}\left(10^{\prime}\right)$ & $54.55(14)$ \\
\hline $\mathrm{C}\left(5^{\prime}\right)-\operatorname{Zr}\left(1^{\prime}\right)-\mathrm{C}\left(1^{\prime}\right)$ & $33.33(15)$ & $\mathrm{C}\left(9^{\prime}\right)-\mathrm{Zr}\left(1^{\prime}\right)-\mathrm{C}\left(10^{\prime}\right)$ & $32.09(14)$ \\
\hline $\mathrm{H}\left(1 \mathrm{M}^{\prime}\right)-\mathrm{Zr}\left(1^{\prime}\right)-\mathrm{C}\left(8^{\prime}\right)$ & $131.4(17)$ & $\mathrm{C}\left(2^{\prime}\right)-\mathrm{Zr}\left(1^{\prime}\right)-\mathrm{C}\left(10^{\prime}\right)$ & $136.25(14)$ \\
\hline $\mathrm{C}\left(11^{\prime}\right)-\mathrm{Zr}\left(1^{\prime}\right)-\mathrm{C}\left(8^{\prime}\right)$ & $106.06(16)$ & $\mathrm{C}\left(7^{\prime}\right)-\mathrm{Zr}\left(1^{\prime}\right)-\mathrm{C}\left(10^{\prime}\right)$ & $53.29(13)$ \\
\hline $\mathrm{C}\left(5^{\prime}\right)-\operatorname{Zr}\left(1^{\prime}\right)-\mathrm{C}\left(8^{\prime}\right)$ & $92.67(14)$ & $\mathrm{C}\left(4^{\prime}\right)-\mathrm{Zr}\left(1^{\prime}\right)-\mathrm{C}\left(10^{\prime}\right)$ & $106.80(14)$ \\
\hline $\mathrm{C}\left(1^{\prime}\right)-\operatorname{Zr}\left(1^{\prime}\right)-\mathrm{C}\left(8^{\prime}\right)$ & $122.68(14)$ & $\mathrm{H}\left(1 \mathrm{M}^{\prime}\right)-\mathrm{Zr}\left(1^{\prime}\right)-\mathrm{C}\left(6^{\prime}\right)$ & $81.4(17)$ \\
\hline $\mathrm{H}\left(1 \mathrm{M}^{\prime}\right)-\mathrm{Zr}\left(1^{\prime}\right)-\mathrm{C}\left(9^{\prime}\right)$ & 105.1(17) & $\mathrm{C}\left(11^{\prime}\right)-\operatorname{Zr}\left(1^{\prime}\right)-\mathrm{C}\left(6^{\prime}\right)$ & $95.12(14)$ \\
\hline $\mathrm{C}\left(11^{\prime}\right)-\mathrm{Zr}\left(1^{\prime}\right)-\mathrm{C}\left(9^{\prime}\right)$ & $137.03(15)$ & $\mathrm{C}\left(5^{\prime}\right)-\mathrm{Zr}\left(1^{\prime}\right)-\mathrm{C}\left(6^{\prime}\right)$ & $147.80(15)$ \\
\hline $\mathrm{C}\left(5^{\prime}\right)-\operatorname{Zr}\left(1^{\prime}\right)-\mathrm{C}\left(9^{\prime}\right)$ & $101.14(15)$ & $\mathrm{C}\left(1^{\prime}\right)-\operatorname{Zr}\left(1^{\prime}\right)-\mathrm{C}\left(6^{\prime}\right)$ & $164.96(13)$ \\
\hline $\mathrm{C}\left(1^{\prime}\right)-\operatorname{Zr}\left(1^{\prime}\right)-\mathrm{C}\left(9^{\prime}\right)$ & $134.00(14)$ & $\mathrm{C}\left(8^{\prime}\right)-\mathrm{Zr}\left(1^{\prime}\right)-\mathrm{C}\left(6^{\prime}\right)$ & $55.20(14)$ \\
\hline $\mathrm{C}\left(8^{\prime}\right)-\operatorname{Zr}\left(1^{\prime}\right)-\mathrm{C}\left(9^{\prime}\right)$ & $33.40(13)$ & $\mathrm{C}\left(9^{\prime}\right)-\operatorname{Zr}\left(1^{\prime}\right)-\mathrm{C}\left(6^{\prime}\right)$ & $54.23(13)$ \\
\hline $\mathrm{H}\left(1 \mathrm{M}^{\prime}\right)-\mathrm{Zr}\left(1^{\prime}\right)-\mathrm{C}\left(2^{\prime}\right)$ & $75.8(17)$ & $\mathrm{C}\left(2^{\prime}\right)-\mathrm{Zr}\left(1^{\prime}\right)-\mathrm{C}\left(6^{\prime}\right)$ & $157.11(14)$ \\
\hline $\mathrm{C}\left(11^{\prime}\right)-\mathrm{Zr}\left(1^{\prime}\right)-\mathrm{C}\left(2^{\prime}\right)$ & $89.77(15)$ & $C\left(7^{\prime}\right)-\operatorname{Zr}\left(1^{\prime}\right)-C\left(6^{\prime}\right)$ & $32.41(13)$ \\
\hline $\mathrm{C}\left(5^{\prime}\right)-\operatorname{Zr}\left(1^{\prime}\right)-\mathrm{C}\left(2^{\prime}\right)$ & $53.93(15)$ & $\mathrm{C}\left(4^{\prime}\right)-\mathrm{Zr}\left(1^{\prime}\right)-\mathrm{C}\left(6^{\prime}\right)$ & $136.53(14)$ \\
\hline $\mathrm{C}\left(1^{\prime}\right)-\operatorname{Zr}\left(1^{\prime}\right)-\mathrm{C}\left(2^{\prime}\right)$ & $33.05(14)$ & $\mathrm{C}\left(10^{\prime}\right)-\operatorname{Zr}\left(1^{\prime}\right)-\mathrm{C}\left(6^{\prime}\right)$ & $32.66(13)$ \\
\hline $\mathrm{C}\left(8^{\prime}\right)-\operatorname{Zr}\left(1^{\prime}\right)-\mathrm{C}\left(2^{\prime}\right)$ & $143.96(14)$ & $\mathrm{H}\left(1 \mathrm{M}^{\prime}\right)-\mathrm{Zr}\left(1^{\prime}\right)-\mathrm{C}\left(3^{\prime}\right)$ & $78.7(17)$ \\
\hline $\mathrm{C}\left(9^{\prime}\right)-\operatorname{Zr}\left(1^{\prime}\right)-\mathrm{C}\left(2^{\prime}\right)$ & $130.71(13)$ & $C\left(11^{\prime}\right)-\operatorname{Zr}\left(1^{\prime}\right)-C\left(3^{\prime}\right)$ & $122.12(14)$ \\
\hline $\mathrm{H}\left(1 \mathrm{M}^{\prime}\right)-\mathrm{Zr}\left(1^{\prime}\right)-\mathrm{C}\left(7^{\prime}\right)$ & $113.0(17)$ & $\mathrm{C}\left(5^{\prime}\right)-\operatorname{Zr}\left(1^{\prime}\right)-\mathrm{C}\left(3^{\prime}\right)$ & $54.41(14)$ \\
\hline $\mathrm{C}\left(11^{\prime}\right)-\mathrm{Zr}\left(1^{\prime}\right)-\mathrm{C}\left(7^{\prime}\right)$ & $83.87(15)$ & $\mathrm{C}\left(1^{\prime}\right)-\operatorname{Zr}\left(1^{\prime}\right)-\mathrm{C}\left(3^{\prime}\right)$ & $55.44(13)$ \\
\hline$C\left(5^{\prime}\right)-\operatorname{Zr}\left(1^{\prime}\right)-C\left(7^{\prime}\right)$ & $118.06(14)$ & $\mathrm{C}\left(8^{\prime}\right)-\mathrm{Zr}\left(1^{\prime}\right)-\mathrm{C}\left(3^{\prime}\right)$ & 119.21(14) \\
\hline$C\left(1^{\prime}\right)-\operatorname{Zr}\left(1^{\prime}\right)-C\left(7^{\prime}\right)$ & $136.71(14)$ & $\mathrm{C}\left(9^{\prime}\right)-\mathrm{Zr}\left(1^{\prime}\right)-\mathrm{C}\left(3^{\prime}\right)$ & $98.05(13)$ \\
\hline$C\left(8^{\prime}\right)-\operatorname{Zr}\left(1^{\prime}\right)-C\left(7^{\prime}\right)$ & $33.08(13)$ & $\mathrm{C}\left(2^{\prime}\right)-\mathrm{Zr}\left(1^{\prime}\right)-\mathrm{C}\left(3^{\prime}\right)$ & $32.82(13)$ \\
\hline $\mathrm{C}\left(9^{\prime}\right)-\operatorname{Zr}\left(1^{\prime}\right)-\mathrm{C}\left(7^{\prime}\right)$ & $53.85(13)$ & $\mathrm{C}\left(7^{\prime}\right)-\mathrm{Zr}\left(1^{\prime}\right)-\mathrm{C}\left(3^{\prime}\right)$ & $150.94(14)$ \\
\hline$C\left(2^{\prime}\right)-\operatorname{Zr}\left(1^{\prime}\right)-C\left(7^{\prime}\right)$ & $169.75(13)$ & $\mathrm{C}\left(4^{\prime}\right)-\mathrm{Zr}\left(1^{\prime}\right)-\mathrm{C}\left(3^{\prime}\right)$ & $32.08(14)$ \\
\hline $\mathrm{H}\left(1 \mathrm{M}^{\prime}\right)-\mathrm{Zr}\left(1^{\prime}\right)-\mathrm{C}\left(4^{\prime}\right)$ & 109.7(17) & $\mathrm{C}\left(10^{\prime}\right)-\mathrm{Zr}\left(1^{\prime}\right)-\mathrm{C}\left(3^{\prime}\right)$ & $108.04(13)$ \\
\hline $\mathrm{C}\left(11^{\prime}\right)-\mathrm{Zr}\left(1^{\prime}\right)-\mathrm{C}\left(4^{\prime}\right)$ & $123.14(15)$ & $\mathrm{C}\left(6^{\prime}\right)-\mathrm{Zr}\left(1^{\prime}\right)-\mathrm{C}\left(3^{\prime}\right)$ & $139.58(13)$ \\
\hline$C\left(5^{\prime}\right)-\operatorname{Zr}\left(1^{\prime}\right)-C\left(4^{\prime}\right)$ & $32.75(14)$ & $\mathrm{C}\left(11^{\prime}\right)-\mathrm{Si}\left(1^{\prime}\right)-\mathrm{C}\left(12^{\prime}\right)$ & $113.5(3)$ \\
\hline$C\left(1^{\prime}\right)-\operatorname{Zr}\left(1^{\prime}\right)-C\left(4^{\prime}\right)$ & $54.79(14)$ & $\mathrm{C}\left(11^{\prime}\right)-\mathrm{Si}\left(1^{\prime}\right)-\mathrm{C}\left(13^{\prime}\right)$ & $116.9(2)$ \\
\hline$C\left(8^{\prime}\right)-\operatorname{Zr}\left(1^{\prime}\right)-C\left(4^{\prime}\right)$ & $91.71(14)$ & $\mathrm{C}\left(12^{\prime}\right)-\mathrm{Si}\left(1^{\prime}\right)-\mathrm{C}\left(13^{\prime}\right)$ & $109.3(2)$ \\
\hline$C\left(9^{\prime}\right)-\operatorname{Zr}\left(1^{\prime}\right)-C\left(4^{\prime}\right)$ & $82.47(14)$ & $\mathrm{C}\left(11^{\prime}\right)-\mathrm{Si}\left(1^{\prime}\right)-\mathrm{C}\left(1^{\prime}\right)$ & $95.66(18)$ \\
\hline
\end{tabular}




\begin{tabular}{|c|c|c|c|}
\hline$C\left(12^{\prime}\right)-\operatorname{Si}\left(1^{\prime}\right)-C\left(1^{\prime}\right)$ & $112.5(2)$ & $\mathrm{C}\left(4^{\prime}\right)-\mathrm{C}\left(3^{\prime}\right)-\mathrm{Si}\left(2^{\prime}\right)$ & $127.2(3)$ \\
\hline$C\left(13^{\prime}\right)-\operatorname{Si}\left(1^{\prime}\right)-C\left(1^{\prime}\right)$ & $108.2(2)$ & $\mathrm{C}\left(2^{\prime}\right)-\mathrm{C}\left(3^{\prime}\right)-\mathrm{Si}\left(2^{\prime}\right)$ & 127.2(3) \\
\hline $\mathrm{C}\left(11^{\prime}\right)-\operatorname{Si}\left(1^{\prime}\right)-\operatorname{Zr}\left(1^{\prime}\right)$ & $44.48(13)$ & $C\left(4^{\prime}\right)-C\left(3^{\prime}\right)-\operatorname{Zr}\left(1^{\prime}\right)$ & $73.1(2)$ \\
\hline $\mathrm{C}\left(12^{\prime}\right)-\operatorname{Si}\left(1^{\prime}\right)-\operatorname{Zr}\left(1^{\prime}\right)$ & $124.21(19)$ & $C\left(2^{\prime}\right)-C\left(3^{\prime}\right)-\operatorname{Zr}\left(1^{\prime}\right)$ & $71.3(2)$ \\
\hline$C\left(13^{\prime}\right)-\operatorname{Si}\left(1^{\prime}\right)-\operatorname{Zr}\left(1^{\prime}\right)$ & $126.40(16)$ & $\operatorname{Si}\left(2^{\prime}\right)-C\left(3^{\prime}\right)-Z r\left(1^{\prime}\right)$ & $125.58(19)$ \\
\hline$C\left(1^{\prime}\right)-\operatorname{Si}\left(1^{\prime}\right)-\operatorname{Zr}\left(1^{\prime}\right)$ & $51.21(12)$ & $\mathrm{C}\left(3^{\prime}\right)-\mathrm{C}\left(4^{\prime}\right)-\mathrm{C}\left(5^{\prime}\right)$ & $109.2(4)$ \\
\hline$C\left(16^{\prime}\right)-\operatorname{Si}\left(2^{\prime}\right)-C\left(15^{\prime}\right)$ & $110.0(3)$ & $\mathrm{C}\left(3^{\prime}\right)-\mathrm{C}\left(4^{\prime}\right)-\operatorname{Zr}\left(1^{\prime}\right)$ & $74.9(2)$ \\
\hline $\mathrm{C}\left(16^{\prime}\right)-\mathrm{Si}\left(2^{\prime}\right)-\mathrm{C}\left(14^{\prime}\right)$ & $109.4(3)$ & $\mathrm{C}\left(5^{\prime}\right)-\mathrm{C}\left(4^{\prime}\right)-\operatorname{Zr}\left(1^{\prime}\right)$ & $70.9(2)$ \\
\hline $\mathrm{C}\left(15^{\prime}\right)-\mathrm{Si}\left(2^{\prime}\right)-\mathrm{C}\left(14^{\prime}\right)$ & $110.9(3)$ & $\mathrm{C}\left(4^{\prime}\right)-\mathrm{C}\left(5^{\prime}\right)-\mathrm{C}\left(1^{\prime}\right)$ & $109.4(4)$ \\
\hline$C\left(16^{\prime}\right)-\operatorname{Si}\left(2^{\prime}\right)-C\left(3^{\prime}\right)$ & $109.6(2)$ & $C\left(4^{\prime}\right)-C\left(5^{\prime}\right)-\operatorname{Zr}\left(1^{\prime}\right)$ & $76.4(2)$ \\
\hline$C\left(15^{\prime}\right)-\operatorname{Si}\left(2^{\prime}\right)-C\left(3^{\prime}\right)$ & $109.8(2)$ & $C\left(1^{\prime}\right)-C\left(5^{\prime}\right)-\operatorname{Zr}\left(1^{\prime}\right)$ & $74.1(2)$ \\
\hline$C\left(14^{\prime}\right)-\operatorname{Si}\left(2^{\prime}\right)-C\left(3^{\prime}\right)$ & 107.1(2) & $\mathrm{C}\left(7^{\prime}\right)-\mathrm{C}\left(6^{\prime}\right)-\mathrm{C}\left(10^{\prime}\right)$ & $105.5(4)$ \\
\hline$C\left(8^{\prime}\right)-\operatorname{Si}\left(3^{\prime}\right)-C\left(17^{\prime}\right)$ & $109.9(2)$ & $C\left(7^{\prime}\right)-C\left(6^{\prime}\right)-\operatorname{Si}\left(4^{\prime}\right)$ & $127.6(3)$ \\
\hline$C\left(8^{\prime}\right)-\operatorname{Si}\left(3^{\prime}\right)-C\left(19^{\prime}\right)$ & $106.7(2)$ & $\mathrm{C}\left(10^{\prime}\right)-\mathrm{C}\left(6^{\prime}\right)-\mathrm{Si}\left(4^{\prime}\right)$ & $126.5(3)$ \\
\hline $\mathrm{C}\left(17^{\prime}\right)-\mathrm{Si}\left(3^{\prime}\right)-\mathrm{C}\left(19^{\prime}\right)$ & $109.2(2)$ & $C\left(7^{\prime}\right)-C\left(6^{\prime}\right)-\operatorname{Zr}\left(1^{\prime}\right)$ & $72.3(2)$ \\
\hline$C\left(8^{\prime}\right)-\operatorname{Si}\left(3^{\prime}\right)-C\left(18^{\prime}\right)$ & $111.4(2)$ & $\mathrm{C}\left(10^{\prime}\right)-\mathrm{C}\left(6^{\prime}\right)-\operatorname{Zr}\left(1^{\prime}\right)$ & $73.0(2)$ \\
\hline $\mathrm{C}\left(17^{\prime}\right)-\mathrm{Si}\left(3^{\prime}\right)-\mathrm{C}\left(18^{\prime}\right)$ & $110.7(2)$ & $\operatorname{Si}\left(4^{\prime}\right)-C\left(6^{\prime}\right)-\operatorname{Zr}\left(1^{\prime}\right)$ & $125.2(2)$ \\
\hline $\mathrm{C}\left(19^{\prime}\right)-\mathrm{Si}\left(3^{\prime}\right)-\mathrm{C}\left(18^{\prime}\right)$ & $108.9(3)$ & $\mathrm{C}\left(6^{\prime}\right)-\mathrm{C}\left(7^{\prime}\right)-\mathrm{C}\left(8^{\prime}\right)$ & $110.8(4)$ \\
\hline $\mathrm{C}\left(20^{\prime}\right)-\mathrm{Si}\left(4^{\prime}\right)-\mathrm{C}\left(21^{\prime}\right)$ & $111.8(3)$ & $\mathrm{C}\left(6^{\prime}\right)-\mathrm{C}\left(7^{\prime}\right)-\operatorname{Zr}\left(1^{\prime}\right)$ & $75.3(2)$ \\
\hline $\mathrm{C}\left(20^{\prime}\right)-\mathrm{Si}\left(4^{\prime}\right)-\mathrm{C}\left(22^{\prime}\right)$ & $107.7(3)$ & $\mathrm{C}\left(8^{\prime}\right)-\mathrm{C}\left(7^{\prime}\right)-\operatorname{Zr}\left(1^{\prime}\right)$ & $72.6(2)$ \\
\hline$C\left(21^{\prime}\right)-\operatorname{Si}\left(4^{\prime}\right)-C\left(22^{\prime}\right)$ & $109.0(3)$ & $\mathrm{C}\left(7^{\prime}\right)-\mathrm{C}\left(8^{\prime}\right)-\mathrm{C}\left(9^{\prime}\right)$ & 105.1(4) \\
\hline$C\left(20^{\prime}\right)-\operatorname{Si}\left(4^{\prime}\right)-C\left(6^{\prime}\right)$ & $110.4(2)$ & $\mathrm{C}\left(7^{\prime}\right)-\mathrm{C}\left(8^{\prime}\right)-\mathrm{Si}\left(3^{\prime}\right)$ & $125.6(3)$ \\
\hline$C\left(21^{\prime}\right)-\operatorname{Si}\left(4^{\prime}\right)-C\left(6^{\prime}\right)$ & $109.6(2)$ & $\mathrm{C}\left(9^{\prime}\right)-\mathrm{C}\left(8^{\prime}\right)-\mathrm{Si}\left(3^{\prime}\right)$ & $127.9(3)$ \\
\hline$C\left(22^{\prime}\right)-\operatorname{Si}\left(4^{\prime}\right)-C\left(6^{\prime}\right)$ & $108.3(2)$ & $C\left(7^{\prime}\right)-C\left(8^{\prime}\right)-\operatorname{Zr}\left(1^{\prime}\right)$ & $74.4(2)$ \\
\hline $\mathrm{C}\left(2^{\prime}\right)-\mathrm{C}\left(1^{\prime}\right)-\mathrm{C}\left(5^{\prime}\right)$ & $105.0(4)$ & $\mathrm{C}\left(9^{\prime}\right)-\mathrm{C}\left(8^{\prime}\right)-\operatorname{Zr}\left(1^{\prime}\right)$ & $73.5(2)$ \\
\hline$C\left(2^{\prime}\right)-C\left(1^{\prime}\right)-\operatorname{Si}\left(1^{\prime}\right)$ & $122.9(3)$ & $\operatorname{Si}\left(3^{\prime}\right)-C\left(8^{\prime}\right)-\operatorname{Zr}\left(1^{\prime}\right)$ & $127.37(19)$ \\
\hline$C\left(5^{\prime}\right)-\mathrm{C}\left(1^{\prime}\right)-\mathrm{Si}\left(1^{\prime}\right)$ & $123.7(3)$ & $\mathrm{C}\left(10^{\prime}\right)-\mathrm{C}\left(9^{\prime}\right)-\mathrm{C}\left(8^{\prime}\right)$ & $109.2(4)$ \\
\hline$C\left(2^{\prime}\right)-C\left(1^{\prime}\right)-\operatorname{Zr}\left(1^{\prime}\right)$ & $74.0(2)$ & $\mathrm{C}\left(10^{\prime}\right)-\mathrm{C}\left(9^{\prime}\right)-\operatorname{Zr}\left(1^{\prime}\right)$ & $75.4(2)$ \\
\hline$C\left(5^{\prime}\right)-C\left(1^{\prime}\right)-\operatorname{Zr}\left(1^{\prime}\right)$ & $72.5(2)$ & $\mathrm{C}\left(8^{\prime}\right)-\mathrm{C}\left(9^{\prime}\right)-\operatorname{Zr}\left(1^{\prime}\right)$ & $73.1(2)$ \\
\hline $\operatorname{Si}\left(1^{\prime}\right)-C\left(1^{\prime}\right)-\operatorname{Zr}\left(1^{\prime}\right)$ & $92.59(15)$ & $\mathrm{C}\left(9^{\prime}\right)-\mathrm{C}\left(10^{\prime}\right)-\mathrm{C}\left(6^{\prime}\right)$ & 109.3(4) \\
\hline $\mathrm{C}\left(1^{\prime}\right)-\mathrm{C}\left(2^{\prime}\right)-\mathrm{C}\left(3^{\prime}\right)$ & $111.0(4)$ & $\mathrm{C}\left(9^{\prime}\right)-\mathrm{C}\left(10^{\prime}\right)-\operatorname{Zr}\left(1^{\prime}\right)$ & $72.5(2)$ \\
\hline$C\left(1^{\prime}\right)-C\left(2^{\prime}\right)-\operatorname{Zr}\left(1^{\prime}\right)$ & $73.0(2)$ & $\mathrm{C}\left(6^{\prime}\right)-\mathrm{C}\left(10^{\prime}\right)-\operatorname{Zr}\left(1^{\prime}\right)$ & $74.4(2)$ \\
\hline$C\left(3^{\prime}\right)-C\left(2^{\prime}\right)-\operatorname{Zr}\left(1^{\prime}\right)$ & $75.9(2)$ & $\mathrm{Si}\left(1^{\prime}\right)-\mathrm{C}\left(11^{\prime}\right)-\operatorname{Zr}\left(1^{\prime}\right)$ & $101.00(19)$ \\
\hline $\mathrm{C}\left(4^{\prime}\right)-\mathrm{C}\left(3^{\prime}\right)-\mathrm{C}\left(2^{\prime}\right)$ & $105.3(4)$ & & \\
\hline
\end{tabular}




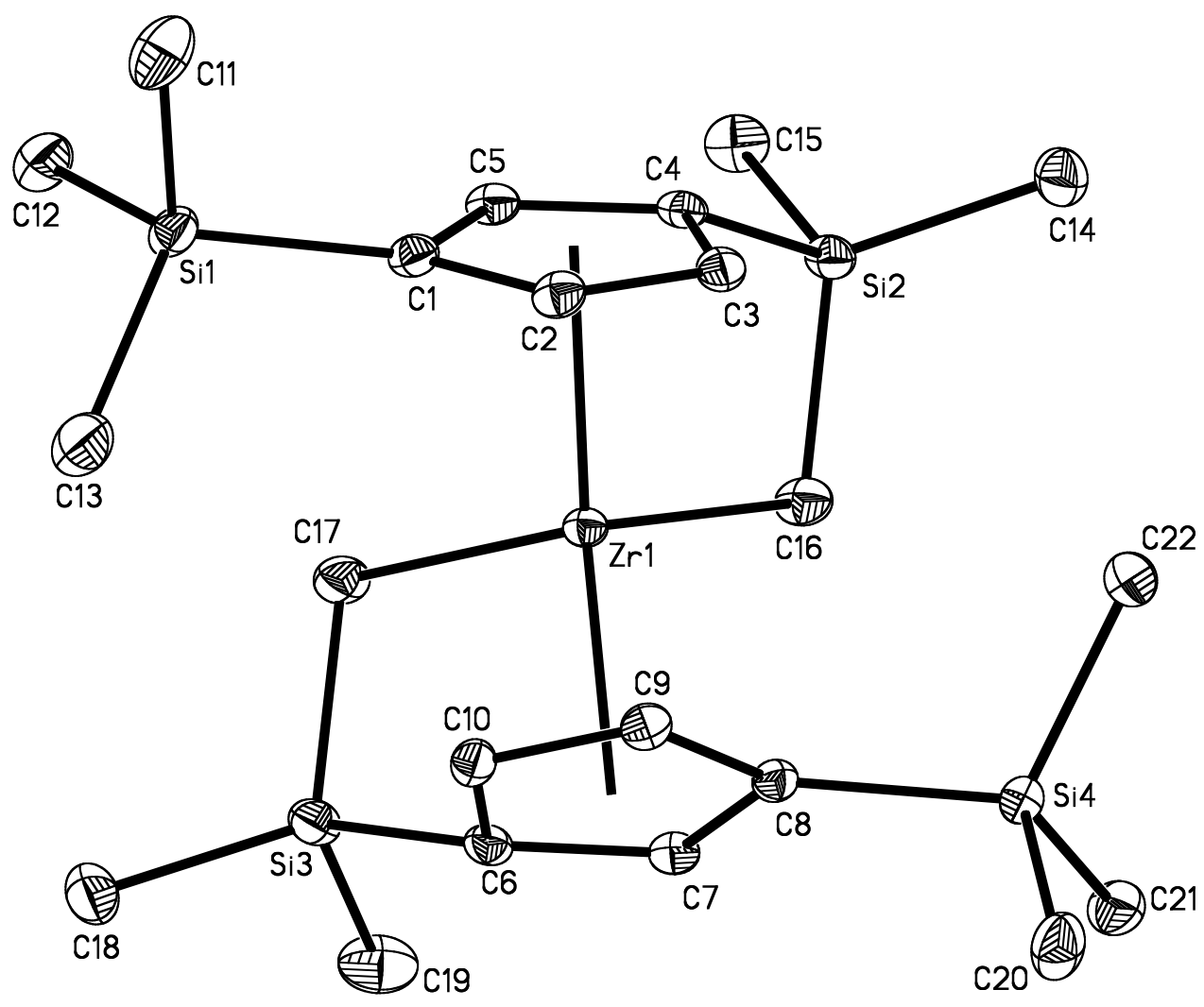

Figure S3. Fully labeled view of the molecular structure of $\mathbf{5}$ at $30 \%$ probability ellipsoids. Hydrogen atoms omitted for clarity. 
Table S3. Crystal Data and Structure Refinement for 5.

Identification code

Empirical formula

Formula weight

Temperature

Wavelength

Crystal system

Space group

Unit cell dimensions

Volume

$\mathrm{Z}$

Density (calculated)

Absorption coefficient

$\mathrm{F}(000)$

Crystal size

Theta range for data collection

Index ranges

Reflections collected

Independent reflections

Completeness to theta $=30.51^{\circ}$

Absorption correction

Max. and min. transmission

Refinement method

Data / restraints / parameters

Goodness-of-fit on $\mathrm{F}^{2}$

Final $\mathrm{R}$ indices [I $>2 \operatorname{sigma}(\mathrm{I})]$

$\mathrm{R}$ indices (all data)

Largest diff. peak and hole jd25

C22 H40 Si4 Zr

508.12

173(2) K

$0.71073 \AA$

Monoclinic

$\mathrm{C} 2 / \mathrm{c}$

$\mathrm{a}=29.984(7) \AA$

$\alpha=90^{\circ}$.

$\mathrm{b}=9.152(2) \AA$

$\beta=108.594(5)^{\circ}$.

$\mathrm{c}=20.813(5) \AA$

$\gamma=90^{\circ}$.

8

$1.247 \mathrm{Mg} / \mathrm{m}^{3}$

$0.589 \mathrm{~mm}^{-1}$

2144

$0.40 \times 0.40 \times 0.10 \mathrm{~mm}^{3}$

2.06 to $30.51^{\circ}$.

$-42<=\mathrm{h}<=22,-13<=\mathrm{k}<=10,-29<=\mathrm{l}<=28$

15226

$8098[\mathrm{R}(\mathrm{int})=0.0289]$

$97.9 \%$

\section{SADABS}

0.9434 and 0.7984

Full-matrix least-squares on $\mathrm{F}^{2}$

8098 / 0 / 404

1.066

$\mathrm{R} 1=0.0370, \mathrm{wR} 2=0.0776$

$\mathrm{R} 1=0.0557, \mathrm{wR} 2=0.0838$

0.803 and -0.469 e. $\AA^{-3}$ 
Table S4. Bond Lengths $[\AA ̊]$ and Angles $\left[{ }^{\circ}\right]$ for 5 .

\begin{tabular}{|c|c|c|c|}
\hline $\operatorname{Zr}(1)-C(17)$ & $2.316(2)$ & $C(6)-C(7)$ & $1.417(3)$ \\
\hline $\operatorname{Zr}(1)-C(16)$ & $2.320(2)$ & $C(6)-C(10)$ & $1.439(3)$ \\
\hline $\mathrm{Zr}(1)-\mathrm{C}(3)$ & $2.4690(19)$ & $\mathrm{C}(7)-\mathrm{C}(8)$ & $1.434(3)$ \\
\hline $\operatorname{Zr}(1)-C(10)$ & $2.4713(19)$ & $\mathrm{C}(8)-\mathrm{C}(9)$ & $1.422(3)$ \\
\hline $\operatorname{Zr}(1)-C(4)$ & $2.4973(18)$ & $\mathrm{C}(9)-\mathrm{C}(10)$ & $1.412(3)$ \\
\hline $\mathrm{Zr}(1)-\mathrm{C}(6)$ & $2.4974(19)$ & & \\
\hline $\operatorname{Zr}(1)-C(7)$ & $2.531(2)$ & $\mathrm{C}(17)-\mathrm{Zr}(1)-\mathrm{C}(16)$ & 98.75(9) \\
\hline $\operatorname{Zr}(1)-C(5)$ & $2.534(2)$ & $\mathrm{C}(17)-\mathrm{Zr}(1)-\mathrm{C}(3)$ & $137.13(7)$ \\
\hline $\operatorname{Zr}(1)-C(9)$ & $2.5471(19)$ & $C(16)-\operatorname{Zr}(1)-C(3)$ & $88.46(7)$ \\
\hline $\operatorname{Zr}(1)-C(2)$ & $2.550(2)$ & $C(17)-\operatorname{Zr}(1)-C(10)$ & $88.53(7)$ \\
\hline $\operatorname{Zr}(1)-C(8)$ & $2.5936(19)$ & $C(16)-\operatorname{Zr}(1)-C(10)$ & $136.89(7)$ \\
\hline $\operatorname{Zr}(1)-C(1)$ & $2.6021(19)$ & $C(3)-Z r(1)-C(10)$ & $114.42(7)$ \\
\hline $\operatorname{Si}(1)-C(12)$ & $1.855(3)$ & $\mathrm{C}(17)-\mathrm{Zr}(1)-\mathrm{C}(4)$ & $110.43(7)$ \\
\hline $\operatorname{Si}(1)-C(11)$ & $1.861(3)$ & $C(16)-Z r(1)-C(4)$ & 70.44(7) \\
\hline $\mathrm{Si}(1)-\mathrm{C}(13)$ & $1.863(3)$ & $C(3)-Z r(1)-C(4)$ & $33.50(6)$ \\
\hline $\operatorname{Si}(1)-C(1)$ & $1.868(2)$ & $C(10)-\operatorname{Zr}(1)-C(4)$ & $145.44(6)$ \\
\hline $\operatorname{Si}(2)-C(16)$ & $1.844(2)$ & $C(17)-Z r(1)-C(6)$ & $70.17(7)$ \\
\hline $\operatorname{Si}(2)-C(15)$ & $1.861(2)$ & $C(16)-Z r(1)-C(6)$ & $110.23(7)$ \\
\hline $\operatorname{Si}(2)-C(14)$ & $1.863(3)$ & $C(3)-Z r(1)-C(6)$ & $145.63(6)$ \\
\hline $\operatorname{Si}(2)-C(4)$ & $1.880(2)$ & $C(10)-Z r(1)-C(6)$ & $33.66(6)$ \\
\hline $\operatorname{Si}(3)-C(17)$ & $1.843(2)$ & $C(4)-Z r(1)-C(6)$ & $179.09(6)$ \\
\hline $\operatorname{Si}(3)-C(18)$ & $1.861(3)$ & $C(17)-Z r(1)-C(7)$ & $91.89(7)$ \\
\hline $\operatorname{Si}(3)-C(19)$ & $1.866(3)$ & $\mathrm{C}(16)-\mathrm{Zr}(1)-\mathrm{C}(7)$ & $83.31(7)$ \\
\hline $\operatorname{Si}(3)-C(6)$ & $1.877(2)$ & $C(3)-Z r(1)-C(7)$ & $130.97(7)$ \\
\hline $\operatorname{Si}(4)-C(21)$ & $1.857(2)$ & $\mathrm{C}(10)-\mathrm{Zr}(1)-\mathrm{C}(7)$ & $53.88(7)$ \\
\hline $\operatorname{Si}(4)-C(20)$ & $1.862(3)$ & $C(4)-Z r(1)-C(7)$ & $147.44(7)$ \\
\hline $\mathrm{Si}(4)-\mathrm{C}(22)$ & $1.865(2)$ & $C(6)-Z r(1)-C(7)$ & $32.72(6)$ \\
\hline $\operatorname{Si}(4)-C(8)$ & $1.866(2)$ & $C(17)-Z r(1)-C(5)$ & $83.45(7)$ \\
\hline $\mathrm{C}(1)-\mathrm{C}(2)$ & $1.421(3)$ & $\mathrm{C}(16)-\mathrm{Zr}(1)-\mathrm{C}(5)$ & $92.30(7)$ \\
\hline $\mathrm{C}(1)-\mathrm{C}(5)$ & $1.433(3)$ & $C(3)-Z r(1)-C(5)$ & $53.94(7)$ \\
\hline $\mathrm{C}(2)-\mathrm{C}(3)$ & $1.413(3)$ & $\mathrm{C}(10)-\mathrm{Zr}(1)-\mathrm{C}(5)$ & $130.80(7)$ \\
\hline $\mathrm{C}(3)-\mathrm{C}(4)$ & $1.431(3)$ & $C(4)-Z r(1)-C(5)$ & $32.84(6)$ \\
\hline$C(4)-C(5)$ & $1.423(3)$ & $C(6)-Z r(1)-C(5)$ & $147.21(7)$ \\
\hline
\end{tabular}




\begin{tabular}{|c|c|c|c|}
\hline$C(7)-\operatorname{Zr}(1)-C(5)$ & $173.07(6)$ & $\mathrm{C}(9)-\mathrm{Zr}(1)-\mathrm{C}(1)$ & $104.43(6)$ \\
\hline $\mathrm{C}(17)-\mathrm{Zr}(1)-\mathrm{C}(9)$ & $120.60(7)$ & $C(2)-\operatorname{Zr}(1)-C(1)$ & $32.01(6)$ \\
\hline$C(16)-\operatorname{Zr}(1)-C(9)$ & $118.63(7)$ & $C(8)-Z r(1)-C(1)$ & $133.72(6)$ \\
\hline$C(3)-\operatorname{Zr}(1)-C(9)$ & $91.04(7)$ & $\mathrm{C}(12)-\mathrm{Si}(1)-\mathrm{C}(11)$ & $109.23(14)$ \\
\hline $\mathrm{C}(10)-\operatorname{Zr}(1)-\mathrm{C}(9)$ & $32.65(6)$ & $\mathrm{C}(12)-\mathrm{Si}(1)-\mathrm{C}(13)$ & $110.03(16)$ \\
\hline $\mathrm{C}(4)-\operatorname{Zr}(1)-\mathrm{C}(9)$ & $124.33(7)$ & $\mathrm{C}(11)-\mathrm{Si}(1)-\mathrm{C}(13)$ & $110.27(16)$ \\
\hline$C(6)-\operatorname{Zr}(1)-C(9)$ & $54.84(6)$ & $\mathrm{C}(12)-\mathrm{Si}(1)-\mathrm{C}(1)$ & $109.47(12)$ \\
\hline$C(7)-\operatorname{Zr}(1)-C(9)$ & $53.15(6)$ & $\mathrm{C}(11)-\mathrm{Si}(1)-\mathrm{C}(1)$ & $106.63(12)$ \\
\hline$C(5)-\operatorname{Zr}(1)-C(9)$ & $133.76(6)$ & $\mathrm{C}(13)-\mathrm{Si}(1)-\mathrm{C}(1)$ & $111.14(11)$ \\
\hline$C(17)-Z r(1)-C(2)$ & $118.99(8)$ & $C(16)-S i(2)-C(15)$ & $115.09(12)$ \\
\hline$C(16)-\operatorname{Zr}(1)-C(2)$ & $120.57(7)$ & $C(16)-S i(2)-C(14)$ & $114.72(12)$ \\
\hline$C(3)-\operatorname{Zr}(1)-C(2)$ & $32.65(7)$ & $C(15)-S i(2)-C(14)$ & $110.50(12)$ \\
\hline$C(10)-\operatorname{Zr}(1)-C(2)$ & $91.02(7)$ & $\mathrm{C}(16)-\mathrm{Si}(2)-\mathrm{C}(4)$ & $96.66(9)$ \\
\hline$C(4)-\operatorname{Zr}(1)-C(2)$ & $54.67(6)$ & $\mathrm{C}(15)-\mathrm{Si}(2)-\mathrm{C}(4)$ & $108.96(11)$ \\
\hline$C(6)-\operatorname{Zr}(1)-C(2)$ & $124.47(7)$ & $\mathrm{C}(14)-\mathrm{Si}(2)-\mathrm{C}(4)$ & 109.91(11) \\
\hline$C(7)-\operatorname{Zr}(1)-C(2)$ & $133.82(6)$ & $C(16)-\operatorname{Si}(2)-\operatorname{Zr}(1)$ & $45.71(7)$ \\
\hline$C(5)-\operatorname{Zr}(1)-C(2)$ & $53.11(6)$ & $C(15)-\operatorname{Si}(2)-\operatorname{Zr}(1)$ & $119.50(9)$ \\
\hline $\mathrm{C}(9)-\mathrm{Zr}(1)-\mathrm{C}(2)$ & $80.93(7)$ & $C(14)-\operatorname{Si}(2)-\operatorname{Zr}(1)$ & $129.89(9)$ \\
\hline $\mathrm{C}(17)-\operatorname{Zr}(1)-\mathrm{C}(8)$ & $123.14(7)$ & $\mathrm{C}(4)-\operatorname{Si}(2)-\mathrm{Zr}(1)$ & $51.33(6)$ \\
\hline$C(16)-\operatorname{Zr}(1)-C(8)$ & $87.69(7)$ & $C(17)-\operatorname{Si}(3)-C(18)$ & $115.40(12)$ \\
\hline$C(3)-\operatorname{Zr}(1)-C(8)$ & $99.21(6)$ & $C(17)-\operatorname{Si}(3)-C(19)$ & $115.33(13)$ \\
\hline$C(10)-\operatorname{Zr}(1)-C(8)$ & $54.21(6)$ & $C(18)-\operatorname{Si}(3)-C(19)$ & $109.17(15)$ \\
\hline$C(4)-\operatorname{Zr}(1)-C(8)$ & $124.62(6)$ & $\mathrm{C}(17)-\mathrm{Si}(3)-\mathrm{C}(6)$ & $96.28(9)$ \\
\hline$C(6)-\operatorname{Zr}(1)-C(8)$ & $55.00(6)$ & $\mathrm{C}(18)-\mathrm{Si}(3)-\mathrm{C}(6)$ & $110.62(11)$ \\
\hline$C(7)-\operatorname{Zr}(1)-C(8)$ & $32.48(6)$ & $\mathrm{C}(19)-\mathrm{Si}(3)-\mathrm{C}(6)$ & $109.26(12)$ \\
\hline$C(5)-\operatorname{Zr}(1)-C(8)$ & 153.11(6) & $C(17)-\operatorname{Si}(3)-\operatorname{Zr}(1)$ & $45.43(7)$ \\
\hline $\mathrm{C}(9)-\operatorname{Zr}(1)-\mathrm{C}(8)$ & $32.10(6)$ & $\mathrm{C}(18)-\mathrm{Si}(3)-\mathrm{Zr}(1)$ & $130.33(10)$ \\
\hline $\mathrm{C}(2)-\mathrm{Zr}(1)-\mathrm{C}(8)$ & $104.48(6)$ & $C(19)-\operatorname{Si}(3)-\operatorname{Zr}(1)$ & $120.40(11)$ \\
\hline $\mathrm{C}(17)-\mathrm{Zr}(1)-\mathrm{C}(1)$ & $88.14(8)$ & $\mathrm{C}(6)-\operatorname{Si}(3)-\mathrm{Zr}(1)$ & $51.16(6)$ \\
\hline$C(16)-\operatorname{Zr}(1)-C(1)$ & $123.33(7)$ & $C(21)-\operatorname{Si}(4)-C(20)$ & $109.69(13)$ \\
\hline$C(3)-\operatorname{Zr}(1)-C(1)$ & $54.11(7)$ & $C(21)-\operatorname{Si}(4)-C(22)$ & $109.72(13)$ \\
\hline$C(10)-\operatorname{Zr}(1)-C(1)$ & $99.20(6)$ & $\mathrm{C}(20)-\mathrm{Si}(4)-\mathrm{C}(22)$ & $109.99(13)$ \\
\hline$C(4)-\operatorname{Zr}(1)-C(1)$ & $54.82(6)$ & $\mathrm{C}(21)-\mathrm{Si}(4)-\mathrm{C}(8)$ & $108.36(11)$ \\
\hline$C(6)-\operatorname{Zr}(1)-C(1)$ & $124.69(7)$ & $\mathrm{C}(20)-\mathrm{Si}(4)-\mathrm{C}(8)$ & $106.56(11)$ \\
\hline$C(7)-\operatorname{Zr}(1)-C(1)$ & $153.05(6)$ & $\mathrm{C}(22)-\mathrm{Si}(4)-\mathrm{C}(8)$ & $112.45(10)$ \\
\hline$C(5)-\operatorname{Zr}(1)-C(1)$ & $32.36(6)$ & $\mathrm{C}(2)-\mathrm{C}(1)-\mathrm{C}(5)$ & $105.57(17)$ \\
\hline
\end{tabular}




\begin{tabular}{|c|c|c|c|}
\hline $\mathrm{C}(2)-\mathrm{C}(1)-\mathrm{Si}(1)$ & $127.29(15)$ & $\mathrm{C}(10)-\mathrm{C}(6)-\mathrm{Si}(3)$ & $123.43(15)$ \\
\hline$C(5)-C(1)-\operatorname{Si}(1)$ & $126.13(15)$ & $C(7)-C(6)-\operatorname{Zr}(1)$ & $74.95(11)$ \\
\hline $\mathrm{C}(2)-\mathrm{C}(1)-\mathrm{Zr}(1)$ & $71.98(11)$ & $C(10)-C(6)-\operatorname{Zr}(1)$ & $72.17(11)$ \\
\hline $\mathrm{C}(5)-\mathrm{C}(1)-\operatorname{Zr}(1)$ & $71.22(10)$ & $\operatorname{Si}(3)-C(6)-\operatorname{Zr}(1)$ & $93.00(7)$ \\
\hline $\mathrm{Si}(1)-\mathrm{C}(1)-\operatorname{Zr}(1)$ & $130.22(10)$ & $\mathrm{C}(6)-\mathrm{C}(7)-\mathrm{C}(8)$ & $111.18(18)$ \\
\hline$C(3)-C(2)-C(1)$ & 109.15(18) & $C(6)-C(7)-\operatorname{Zr}(1)$ & $72.33(11)$ \\
\hline$C(3)-C(2)-\operatorname{Zr}(1)$ & $70.53(11)$ & $\mathrm{C}(8)-\mathrm{C}(7)-\operatorname{Zr}(1)$ & $76.15(11)$ \\
\hline$C(1)-C(2)-\operatorname{Zr}(1)$ & $76.02(11)$ & $\mathrm{C}(9)-\mathrm{C}(8)-\mathrm{C}(7)$ & $105.37(17)$ \\
\hline$C(2)-C(3)-C(4)$ & 109.17(18) & $\mathrm{C}(9)-\mathrm{C}(8)-\mathrm{Si}(4)$ & $127.87(15)$ \\
\hline $\mathrm{C}(2)-\mathrm{C}(3)-\operatorname{Zr}(1)$ & $76.83(11)$ & $\mathrm{C}(7)-\mathrm{C}(8)-\mathrm{Si}(4)$ & $125.63(15)$ \\
\hline $\mathrm{C}(4)-\mathrm{C}(3)-\mathrm{Zr}(1)$ & $74.34(11)$ & $\mathrm{C}(9)-\mathrm{C}(8)-\operatorname{Zr}(1)$ & $72.15(10)$ \\
\hline $\mathrm{C}(5)-\mathrm{C}(4)-\mathrm{C}(3)$ & $105.36(17)$ & $\mathrm{C}(7)-\mathrm{C}(8)-\operatorname{Zr}(1)$ & $71.37(10)$ \\
\hline $\mathrm{C}(5)-\mathrm{C}(4)-\mathrm{Si}(2)$ & $123.16(15)$ & $\operatorname{Si}(4)-C(8)-\operatorname{Zr}(1)$ & $130.34(9)$ \\
\hline $\mathrm{C}(3)-\mathrm{C}(4)-\mathrm{Si}(2)$ & $123.44(15)$ & $\mathrm{C}(10)-\mathrm{C}(9)-\mathrm{C}(8)$ & $109.18(17)$ \\
\hline$C(5)-C(4)-\operatorname{Zr}(1)$ & $75.01(11)$ & $C(10)-C(9)-Z r(1)$ & $70.72(11)$ \\
\hline $\mathrm{C}(3)-\mathrm{C}(4)-\mathrm{Zr}(1)$ & $72.17(10)$ & $\mathrm{C}(8)-\mathrm{C}(9)-\operatorname{Zr}(1)$ & $75.75(11)$ \\
\hline $\mathrm{Si}(2)-\mathrm{C}(4)-\operatorname{Zr}(1)$ & $92.69(7)$ & $\mathrm{C}(9)-\mathrm{C}(10)-\mathrm{C}(6)$ & $109.16(18)$ \\
\hline$C(4)-C(5)-C(1)$ & $110.73(18)$ & $C(9)-C(10)-Z r(1)$ & $76.63(11)$ \\
\hline$C(4)-C(5)-Z r(1)$ & $72.15(11)$ & $C(6)-C(10)-Z r(1)$ & $74.17(11)$ \\
\hline$C(1)-C(5)-Z r(1)$ & $76.42(11)$ & $\operatorname{Si}(2)-C(16)-\operatorname{Zr}(1)$ & $99.59(9)$ \\
\hline$C(7)-C(6)-C(10)$ & $105.10(17)$ & $\mathrm{Si}(3)-\mathrm{C}(17)-\operatorname{Zr}(1)$ & $100.05(9)$ \\
\hline$C(7)-C(6)-S i(3)$ & $123.62(15)$ & & \\
\hline
\end{tabular}

Symmetry transformations used to generate equivalent atoms: 


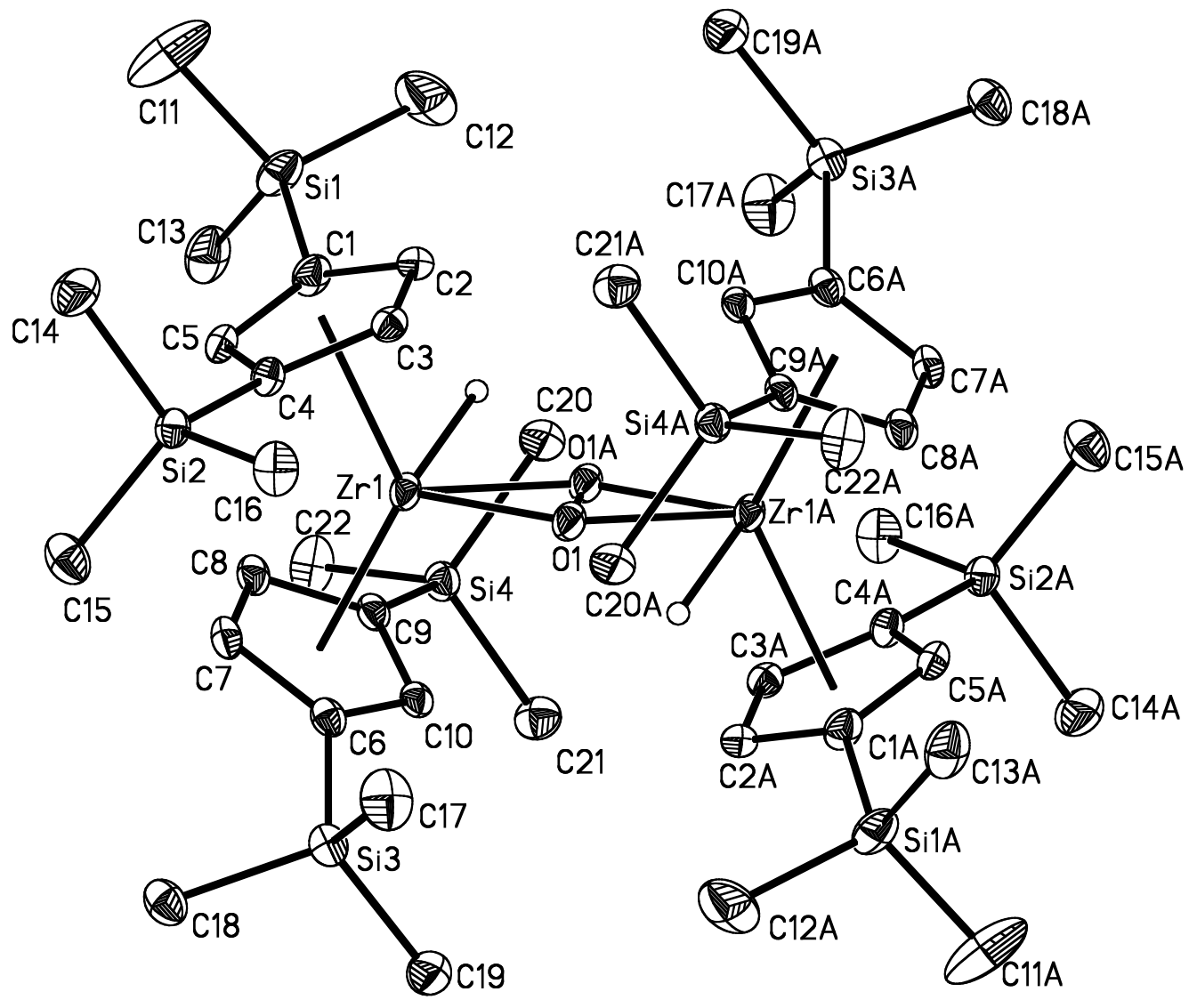

Figure S4. Fully labeled view of the molecular structure of $\mathbf{7}$ at $30 \%$ probability ellipsoids. Hydrogen atoms, except for the zirconium hydrides, omitted for clarity. 
Table S5. Crystal Data and Structure Refinement for $\mathbf{7}$.

Identification code

Empirical formula

Formula weight

Temperature

Wavelength

Crystal system

Space group

Unit cell dimensions

Volume

$\mathrm{Z}$

Density (calculated)

Absorption coefficient

$\mathrm{F}(000)$

Crystal size

Theta range for data collection

Index ranges

Reflections collected

Independent reflections

Completeness to theta $=28.28^{\circ}$

Absorption correction

Refinement method

Data / restraints / parameters

Goodness-of-fit on $\mathrm{F}^{2}$

Final R indices [I $>2 \operatorname{sigma}(\mathrm{I})]$

$\mathrm{R}$ indices (all data)

Largest diff. peak and hole jd21

C22 H43 O0.50 Si4 Zr

519.14

173(2) K

$0.71073 \AA$

Monoclinic

$\mathrm{P} 2(1) / \mathrm{n}$

$\mathrm{a}=11.9916(7) \AA \quad \alpha=90^{\circ}$.

$\mathrm{b}=15.6633(9) \AA \quad \beta=99.528(2)^{\circ}$.

$\mathrm{c}=15.3898(8) \AA \quad \gamma=90^{\circ}$.

2850.8(3) $\AA^{3}$

4

$1.210 \mathrm{Mg} / \mathrm{m}^{3}$

$0.562 \mathrm{~mm}^{-1}$

1100

$0.3 \times 0.25 \times 0.2 \mathrm{~mm}^{3}$

1.87 to $28.28^{\circ}$.

$-15<=\mathrm{h}<=15,-20<=\mathrm{k}<=20,-20<=\mathrm{l}<=20$

19663

$7015[\mathrm{R}(\mathrm{int})=0.0289]$

$99.3 \%$

\section{SADABS}

Full-matrix least-squares on $\mathrm{F}^{2}$

7015 / 0 / 393

1.063

$\mathrm{R} 1=0.0387, \mathrm{wR} 2=0.0823$

$\mathrm{R} 1=0.0523, \mathrm{wR} 2=0.0875$

1.111 and -0.706 e. $\AA^{-3}$ 
Table S6. Bond Lengths $[\AA ̊]$ and Angles $\left[{ }^{\circ}\right]$ for 7.

\begin{tabular}{|c|c|c|c|}
\hline $\mathrm{Zr}(1)-\mathrm{H}(2 \mathrm{M})$ & $1.88(5)$ & $\mathrm{C}(4)-\mathrm{C}(5)$ & $1.419(3)$ \\
\hline $\mathrm{Zr}(1)-\mathrm{H}(1 \mathrm{M})$ & $1.98(6)$ & $\mathrm{C}(6)-\mathrm{C}(10)$ & $1.423(2)$ \\
\hline $\operatorname{Zr}(1)-\mathrm{O}(1)$ & $1.932(3)$ & $\mathrm{C}(6)-\mathrm{C}(7)$ & $1.430(3)$ \\
\hline $\mathrm{Zr}(1)-\mathrm{O}(1) \# 1$ & $1.945(3)$ & $\mathrm{C}(7)-\mathrm{C}(8)$ & $1.398(3)$ \\
\hline $\operatorname{Zr}(1)-\mathrm{C}(2)$ & $2.5216(19)$ & $\mathrm{C}(8)-\mathrm{C}(9)$ & $1.430(3)$ \\
\hline $\operatorname{Zr}(1)-C(3)$ & $2.5248(18)$ & $\mathrm{C}(9)-\mathrm{C}(10)$ & $1.419(3)$ \\
\hline $\operatorname{Zr}(1)-\mathrm{C}(7)$ & $2.5458(18)$ & $\mathrm{H}(2 \mathrm{M})-\mathrm{Zr}(1)-\mathrm{H}(1 \mathrm{M})$ & $158(2)$ \\
\hline $\operatorname{Zr}(1)-C(8)$ & $2.5478(18)$ & $\mathrm{H}(2 \mathrm{M})-\mathrm{Zr}(1)-\mathrm{O}(1)$ & $68.8(14)$ \\
\hline $\operatorname{Zr}(1)-\mathrm{C}(1)$ & $2.556(2)$ & $\mathrm{H}(1 \mathrm{M})-\mathrm{Zr}(1)-\mathrm{O}(1)$ & $90.9(17)$ \\
\hline $\operatorname{Zr}(1)-C(4)$ & $2.5664(18)$ & $\mathrm{H}(2 \mathrm{M})-\mathrm{Zr}(1)-\mathrm{O}(1) \# 1$ & $96.1(14)$ \\
\hline $\mathrm{Zr}(1)-\mathrm{C}(10)$ & $2.5677(18)$ & $\mathrm{H}(1 \mathrm{M})-\mathrm{Zr}(1)-\mathrm{O}(1) \# 1$ & $64.0(17)$ \\
\hline $\operatorname{Zr}(1)-C(5)$ & $2.5709(18)$ & $\mathrm{O}(1)-\mathrm{Zr}(1)-\mathrm{O}(1) \# 1$ & $27.24(16)$ \\
\hline $\mathrm{Si}(1)-\mathrm{C}(13)$ & $1.859(3)$ & $\mathrm{H}(2 \mathrm{M})-\mathrm{Zr}(1)-\mathrm{C}(2)$ & $103.8(15)$ \\
\hline $\mathrm{Si}(1)-\mathrm{C}(12)$ & $1.858(3)$ & $\mathrm{H}(1 \mathrm{M})-\mathrm{Zr}(1)-\mathrm{C}(2)$ & $67.6(18)$ \\
\hline $\mathrm{Si}(1)-\mathrm{C}(11)$ & $1.863(3)$ & $\mathrm{O}(1)-\mathrm{Zr}(1)-\mathrm{C}(2)$ & $92.19(10)$ \\
\hline $\mathrm{Si}(1)-\mathrm{C}(1)$ & $1.865(2)$ & $\mathrm{O}(1) \# 1-\mathrm{Zr}(1)-\mathrm{C}(2)$ & $85.44(10)$ \\
\hline $\mathrm{Si}(2)-\mathrm{C}(15)$ & $1.858(2)$ & $\mathrm{H}(2 \mathrm{M})-\mathrm{Zr}(1)-\mathrm{C}(3)$ & $72.2(15)$ \\
\hline $\mathrm{Si}(2)-\mathrm{C}(16)$ & $1.860(2)$ & $\mathrm{H}(1 \mathrm{M})-\mathrm{Zr}(1)-\mathrm{C}(3)$ & $98.9(18)$ \\
\hline $\mathrm{Si}(2)-\mathrm{C}(14)$ & $1.865(3)$ & $\mathrm{O}(1)-\mathrm{Zr}(1)-\mathrm{C}(3)$ & $85.33(10)$ \\
\hline $\mathrm{Si}(2)-\mathrm{C}(4)$ & $1.8695(18)$ & $\mathrm{O}(1) \# 1-\mathrm{Zr}(1)-\mathrm{C}(3)$ & $93.45(10)$ \\
\hline $\mathrm{Si}(3)-\mathrm{C}(18)$ & $1.865(2)$ & $C(2)-Z r(1)-C(3)$ & $31.97(6)$ \\
\hline $\mathrm{Si}(3)-\mathrm{C}(17)$ & $1.867(3)$ & $\mathrm{H}(2 \mathrm{M})-\mathrm{Zr}(1)-\mathrm{C}(7)$ & $82.7(14)$ \\
\hline $\mathrm{Si}(3)-\mathrm{C}(19)$ & $1.867(2)$ & $\mathrm{H}(1 \mathrm{M})-\mathrm{Zr}(1)-\mathrm{C}(7)$ & 118.1(17) \\
\hline $\mathrm{Si}(3)-\mathrm{C}(6)$ & $1.875(2)$ & $\mathrm{O}(1)-\mathrm{Zr}(1)-\mathrm{C}(7)$ & $131.82(10)$ \\
\hline $\mathrm{Si}(4)-\mathrm{C}(22)$ & $1.861(2)$ & $\mathrm{O}(1) \# 1-\mathrm{Zr}(1)-\mathrm{C}(7)$ & $140.83(10)$ \\
\hline $\mathrm{Si}(4)-\mathrm{C}(20)$ & $1.862(2)$ & $C(2)-\operatorname{Zr}(1)-C(7)$ & 133.07(6) \\
\hline $\mathrm{Si}(4)-\mathrm{C}(21)$ & $1.867(2)$ & $C(3)-Z r(1)-C(7)$ & $122.64(6)$ \\
\hline $\mathrm{Si}(4)-\mathrm{C}(9)$ & $1.8759(19)$ & $\mathrm{H}(2 \mathrm{M})-\mathrm{Zr}(1)-\mathrm{C}(8)$ & $114.5(14)$ \\
\hline $\mathrm{O}(1)-\mathrm{O}(1) \# 1$ & $0.913(6)$ & $\mathrm{H}(1 \mathrm{M})-\mathrm{Zr}(1)-\mathrm{C}(8)$ & $86.3(17)$ \\
\hline $\mathrm{O}(1)-\mathrm{Zr}(1) \# 1$ & $1.945(3)$ & $\mathrm{O}(1)-\mathrm{Zr}(1)-\mathrm{C}(8)$ & $141.44(10)$ \\
\hline$C(1)-C(5)$ & $1.421(3)$ & $\mathrm{O}(1) \# 1-\mathrm{Zr}(1)-\mathrm{C}(8)$ & $129.21(10)$ \\
\hline $\mathrm{C}(1)-\mathrm{C}(2)$ & $1.430(3)$ & $C(2)-\operatorname{Zr}(1)-C(8)$ & 121.64(6) \\
\hline $\mathrm{C}(2)-\mathrm{C}(3)$ & $1.390(3)$ & $C(3)-\operatorname{Zr}(1)-C(8)$ & $133.10(6)$ \\
\hline$C(3)-C(4)$ & $1.427(3)$ & $C(7)-\operatorname{Zr}(1)-C(8)$ & $31.85(6)$ \\
\hline
\end{tabular}




\begin{tabular}{|c|c|c|c|}
\hline $\mathrm{H}(2 \mathrm{M})-\mathrm{Zr}(1)-\mathrm{C}(1)$ & $119.9(15)$ & $C(4)-Z r(1)-C(5)$ & $32.08(6)$ \\
\hline $\mathrm{H}(1 \mathrm{M})-\mathrm{Zr}(1)-\mathrm{C}(1)$ & $64.1(17)$ & $C(10)-\operatorname{Zr}(1)-C(5)$ & $130.82(6)$ \\
\hline $\mathrm{O}(1)-\mathrm{Zr}(1)-\mathrm{C}(1)$ & $123.88(10)$ & $\mathrm{C}(13)-\mathrm{Si}(1)-\mathrm{C}(12)$ & $108.54(13)$ \\
\hline $\mathrm{O}(1) \# 1-\mathrm{Zr}(1)-\mathrm{C}(1)$ & $110.32(10)$ & $\mathrm{C}(13)-\mathrm{Si}(1)-\mathrm{C}(11)$ & $108.70(15)$ \\
\hline $\mathrm{C}(2)-\mathrm{Zr}(1)-\mathrm{C}(1)$ & $32.70(6)$ & $\mathrm{C}(12)-\mathrm{Si}(1)-\mathrm{C}(11)$ & $110.39(17)$ \\
\hline$C(3)-\operatorname{Zr}(1)-C(1)$ & $53.88(6)$ & $\mathrm{C}(13)-\mathrm{Si}(1)-\mathrm{C}(1)$ & $113.95(11)$ \\
\hline$C(7)-\operatorname{Zr}(1)-C(1)$ & $103.82(6)$ & $\mathrm{C}(12)-\mathrm{Si}(1)-\mathrm{C}(1)$ & $110.13(11)$ \\
\hline $\mathrm{C}(8)-\operatorname{Zr}(1)-\mathrm{C}(1)$ & $89.09(6)$ & $\mathrm{C}(11)-\mathrm{Si}(1)-\mathrm{C}(1)$ & $105.07(12)$ \\
\hline $\mathrm{H}(2 \mathrm{M})-\mathrm{Zr}(1)-\mathrm{C}(4)$ & $66.2(15)$ & $C(15)-S i(2)-C(16)$ & $111.50(12)$ \\
\hline $\mathrm{H}(1 \mathrm{M})-\mathrm{Zr}(1)-\mathrm{C}(4)$ & $116.8(18)$ & $\mathrm{C}(15)-\mathrm{Si}(2)-\mathrm{C}(14)$ & $108.25(12)$ \\
\hline $\mathrm{O}(1)-\mathrm{Zr}(1)-\mathrm{C}(4)$ & $110.99(10)$ & $\mathrm{C}(16)-\mathrm{Si}(2)-\mathrm{C}(14)$ & $108.55(13)$ \\
\hline $\mathrm{O}(1) \# 1-\mathrm{Zr}(1)-\mathrm{C}(4)$ & $125.28(10)$ & $\mathrm{C}(15)-\mathrm{Si}(2)-\mathrm{C}(4)$ & $112.00(10)$ \\
\hline$C(2)-\operatorname{Zr}(1)-C(4)$ & $53.67(6)$ & $\mathrm{C}(16)-\mathrm{Si}(2)-\mathrm{C}(4)$ & $110.21(9)$ \\
\hline$C(3)-\operatorname{Zr}(1)-C(4)$ & $32.55(6)$ & $\mathrm{C}(14)-\mathrm{Si}(2)-\mathrm{C}(4)$ & $106.12(11)$ \\
\hline$C(7)-\operatorname{Zr}(1)-C(4)$ & $90.18(6)$ & $\mathrm{C}(18)-\mathrm{Si}(3)-\mathrm{C}(17)$ & $108.78(12)$ \\
\hline $\mathrm{C}(8)-\operatorname{Zr}(1)-\mathrm{C}(4)$ & $104.43(6)$ & $\mathrm{C}(18)-\mathrm{Si}(3)-\mathrm{C}(19)$ & $109.36(11)$ \\
\hline$C(1)-\operatorname{Zr}(1)-C(4)$ & $54.28(6)$ & $\mathrm{C}(17)-\mathrm{Si}(3)-\mathrm{C}(19)$ & $108.02(13)$ \\
\hline $\mathrm{H}(2 \mathrm{M})-\mathrm{Zr}(1)-\mathrm{C}(10)$ & $92.5(15)$ & $\mathrm{C}(18)-\mathrm{Si}(3)-\mathrm{C}(6)$ & $107.56(10)$ \\
\hline $\mathrm{H}(1 \mathrm{M})-\mathrm{Zr}(1)-\mathrm{C}(10)$ & $95.6(18)$ & $\mathrm{C}(17)-\mathrm{Si}(3)-\mathrm{C}(6)$ & $114.99(12)$ \\
\hline $\mathrm{O}(1)-\operatorname{Zr}(1)-\mathrm{C}(10)$ & $89.69(10)$ & $\mathrm{C}(19)-\mathrm{Si}(3)-\mathrm{C}(6)$ & $108.03(10)$ \\
\hline $\mathrm{O}(1) \# 1-\mathrm{Zr}(1)-\mathrm{C}(10)$ & $88.60(10)$ & $C(22)-\operatorname{Si}(4)-C(20)$ & $110.12(13)$ \\
\hline$C(2)-\operatorname{Zr}(1)-C(10)$ & $163.09(6)$ & $C(22)-\operatorname{Si}(4)-C(21)$ & $108.18(12)$ \\
\hline$C(3)-\operatorname{Zr}(1)-C(10)$ & $164.73(6)$ & $C(20)-S i(4)-C(21)$ & $108.49(11)$ \\
\hline$C(7)-\operatorname{Zr}(1)-C(10)$ & $52.53(6)$ & $\mathrm{C}(22)-\mathrm{Si}(4)-\mathrm{C}(9)$ & $108.02(10)$ \\
\hline$C(8)-\operatorname{Zr}(1)-C(10)$ & $52.48(6)$ & $\mathrm{C}(20)-\mathrm{Si}(4)-\mathrm{C}(9)$ & $113.26(11)$ \\
\hline$C(1)-\operatorname{Zr}(1)-C(10)$ & $138.83(6)$ & $\mathrm{C}(21)-\mathrm{Si}(4)-\mathrm{C}(9)$ & $108.66(10)$ \\
\hline$C(4)-\operatorname{Zr}(1)-C(10)$ & $140.18(6)$ & $\mathrm{O}(1) \# 1-\mathrm{O}(1)-\mathrm{Zr}(1)$ & $77.2(4)$ \\
\hline $\mathrm{H}(2 \mathrm{M})-\mathrm{Zr}(1)-\mathrm{C}(5)$ & $95.4(14)$ & $\mathrm{O}(1) \# 1-\mathrm{O}(1)-\mathrm{Zr}(1) \# 1$ & $75.6(4)$ \\
\hline $\mathrm{H}(1 \mathrm{M})-\mathrm{Zr}(1)-\mathrm{C}(5)$ & $94.6(17)$ & $\mathrm{Zr}(1)-\mathrm{O}(1)-\mathrm{Zr}(1) \# 1$ & $152.76(16)$ \\
\hline $\mathrm{O}(1)-\mathrm{Zr}(1)-\mathrm{C}(5)$ & $138.09(10)$ & $\mathrm{C}(5)-\mathrm{C}(1)-\mathrm{C}(2)$ & $105.13(16)$ \\
\hline $\mathrm{O}(1) \# 1-\mathrm{Zr}(1)-\mathrm{C}(5)$ & $138.21(10)$ & $\mathrm{C}(5)-\mathrm{C}(1)-\mathrm{Si}(1)$ & $125.81(14)$ \\
\hline$C(2)-\operatorname{Zr}(1)-C(5)$ & $52.78(6)$ & $\mathrm{C}(2)-\mathrm{C}(1)-\mathrm{Si}(1)$ & $125.23(15)$ \\
\hline$C(3)-\operatorname{Zr}(1)-C(5)$ & $52.77(6)$ & $C(5)-C(1)-\operatorname{Zr}(1)$ & $74.47(11)$ \\
\hline$C(7)-\operatorname{Zr}(1)-C(5)$ & $80.51(6)$ & $\mathrm{C}(2)-\mathrm{C}(1)-\mathrm{Zr}(1)$ & $72.31(11)$ \\
\hline$C(8)-\operatorname{Zr}(1)-C(5)$ & $80.43(6)$ & $\operatorname{Si}(1)-C(1)-\operatorname{Zr}(1)$ & $135.13(10)$ \\
\hline $\mathrm{C}(1)-\operatorname{Zr}(1)-\mathrm{C}(5)$ & $32.19(6)$ & $\mathrm{C}(3)-\mathrm{C}(2)-\mathrm{C}(1)$ & $109.45(16)$ \\
\hline
\end{tabular}




\begin{tabular}{|c|c|}
\hline$C(3)-C(2)-\operatorname{Zr}(1)$ & $74.14(11)$ \\
\hline$C(1)-C(2)-\operatorname{Zr}(1)$ & $74.99(11)$ \\
\hline$C(2)-C(3)-C(4)$ & $109.27(16)$ \\
\hline$C(2)-C(3)-\operatorname{Zr}(1)$ & $73.89(11)$ \\
\hline$C(4)-C(3)-\operatorname{Zr}(1)$ & $75.33(11)$ \\
\hline$C(5)-C(4)-C(3)$ & $105.42(16)$ \\
\hline $\mathrm{C}(5)-\mathrm{C}(4)-\mathrm{Si}(2)$ & $126.04(14)$ \\
\hline $\mathrm{C}(3)-\mathrm{C}(4)-\mathrm{Si}(2)$ & $125.63(14)$ \\
\hline$C(5)-C(4)-\operatorname{Zr}(1)$ & $74.13(10)$ \\
\hline$C(3)-C(4)-\operatorname{Zr}(1)$ & $72.12(10)$ \\
\hline $\operatorname{Si}(2)-C(4)-\operatorname{Zr}(1)$ & $133.52(10)$ \\
\hline$C(4)-C(5)-C(1)$ & $110.70(16)$ \\
\hline$C(4)-C(5)-\operatorname{Zr}(1)$ & $73.79(10)$ \\
\hline$C(1)-C(5)-\operatorname{Zr}(1)$ & $73.34(11)$ \\
\hline$C(10)-C(6)-C(7)$ & $104.97(16)$ \\
\hline$C(10)-C(6)-S i(3)$ & $126.50(15)$ \\
\hline$C(7)-C(6)-S i(3)$ & $126.90(14)$ \\
\hline$C(10)-C(6)-\operatorname{Zr}(1)$ & $73.56(11)$ \\
\hline$C(7)-C(6)-\operatorname{Zr}(1)$ & $72.58(11)$ \\
\hline $\operatorname{Si}(3)-C(6)-\operatorname{Zr}(1)$ & $129.65(9)$ \\
\hline $\mathrm{C}(8)-\mathrm{C}(7)-\mathrm{C}(6)$ & $109.38(16)$ \\
\hline $\mathrm{C}(8)-\mathrm{C}(7)-\mathrm{Zr}(1)$ & $74.15(11)$ \\
\hline$C(6)-C(7)-Z r(1)$ & $75.03(10)$ \\
\hline $\mathrm{C}(7)-\mathrm{C}(8)-\mathrm{C}(9)$ & 109.32(17) \\
\hline$C(7)-C(8)-Z r(1)$ & $74.00(10)$ \\
\hline $\mathrm{C}(9)-\mathrm{C}(8)-\mathrm{Zr}(1)$ & $74.94(10)$ \\
\hline $\mathrm{C}(10)-\mathrm{C}(9)-\mathrm{C}(8)$ & $105.11(16)$ \\
\hline $\mathrm{C}(10)-\mathrm{C}(9)-\mathrm{Si}(4)$ & $126.47(14)$ \\
\hline $\mathrm{C}(8)-\mathrm{C}(9)-\mathrm{Si}(4)$ & $126.96(14)$ \\
\hline$C(10)-C(9)-\operatorname{Zr}(1)$ & $73.62(10)$ \\
\hline $\mathrm{C}(8)-\mathrm{C}(9)-\mathrm{Zr}(1)$ & $72.67(10)$ \\
\hline $\operatorname{Si}(4)-C(9)-Z r(1)$ & 128.99(9) \\
\hline $\mathrm{C}(9)-\mathrm{C}(10)-\mathrm{C}(6)$ & $111.22(16)$ \\
\hline $\mathrm{C}(9)-\mathrm{C}(10)-\mathrm{Zr}(1)$ & $74.36(10)$ \\
\hline$C(6)-C(10)-\operatorname{Zr}(1)$ & $74.33(11)$ \\
\hline
\end{tabular}


Symmetry transformations used to generate equivalent atoms:

$\# 1-\mathrm{x},-\mathrm{y},-\mathrm{z}+1$ 